Revue musicale OICRM

revue musicale oicrm

\title{
Variations... sur Yves Margat (humour estetico)( Anthologie du PHEM » / Musicographes, 2)
}

\section{Federico Lazzaro}

Volume 5, numéro 2, 2018

URI : https://id.erudit.org/iderudit/1054151ar

DOI : https://doi.org/10.7202/1054151ar

Aller au sommaire du numéro

\section{Éditeur(s)}

Observatoire interdisciplinaire de création et recherche en musique (OICRM)

\section{ISSN}

2368-7061 (numérique)

Découvrir la revue

Citer cet article

Lazzaro, F. (2018). Variations... sur Yves Margat (humour estetico)(« Anthologie du PHEM » / Musicographes, 2). Revue musicale OICRM, 5(2), 149-179.

https://doi.org/10.7202/1054151ar
Résumé de l'article

Les 126 « Variations... sans thème » publiées par Yves Margat dans Le Guide du concert entre 1934 et 1939 sont une source précieuse pour l'étude du discours sur la musique en France dans cette période de crise économique, culturelle et politique. Les billets humoristiques de Margat ironisent à la fois sur la place de la musique dans la société parisienne et sur la crise du langage et de l'écoute musicaux. Margat construit sa crédibilité de faiseur d'opinion par son professorat (ses cours d'harmonie sont amplement publicisés par la revue qui publie sa rubrique hebdomadaire) et, réciproquement, promeut son enseignement comme solution aux maux qu'il dénonce, ce qui donne à ses billets un statut digne d'être problématisé. 


\title{
Variations... sur Yves Margat (humour estetico) ( Anthologie du PHEM " / Musicographes, 2)
}

\author{
Federico Lazzaro
}

\begin{abstract}
Résumé
Les 126 "Variations... sans thème " publiées par Yves Margat dans Le Guide du concert entre 1934 et 1939 sont une source précieuse pour l'étude du discours sur la musique en France dans cette période de crise économique, culturelle et politique. Les billets humoristiques de Margat ironisent à la fois sur la place de la musique dans la société parisienne et sur la crise du langage et de l'écoute musicaux. Margat construit sa crédibilité de faiseur d'opinion par son professorat (ses cours d'harmonie sont amplement publicisés par la revue qui publie sa rubrique hebdomadaire) et, réciproquement, promeut son enseignement comme solution aux maux qu'il dénonce, ce qui donne à ses billets un statut digne d'être problématisé.
\end{abstract}

Mots clés : Yves Margat ; crise des années 1930 ; ironie ; modernisme ; idéalisme.

\begin{abstract}
The 126 "Variations... sans thème» (Variations... without theme) which Yves Margat wrote for Le Guide du concert between 1934 and 1939 provide a particularly interesting insight into the discourse about music in France during the economical, cultural and political crisis of that period. Margat's humorous column is ironical about the place of music within the Parisian society as well as about the crisis of musical language and listening. Margat builds his reliability as an opinion-maker by his activity as a teacher (his harmony courses are widely publicized in the journal publishing his column) and, conversely, he promotes his courses as the solution to the ills he speaks out against. This calls into question the status of Margat's columns.
\end{abstract}

Keywords: Yves Margat; crisis of the 1930s; irony; modernism; idealism. 
Les "Variations... sans thème " publiées dans quasiment chaque numéro du Guide du concert entre octobre 1934 et mai 1939 (date à laquelle la revue a interrompu sa parution en raison de la guerre ${ }^{1}$ ) constituent un de ces lieux où le discours esthétique dans la presse musicale se glisse sans que l'on ne s'y attende ${ }^{2}$. En fait, la rubrique d'Yves Margat (1896-1971) prend la forme d'une série de billets qui commentent de façon humoristique les changements sociomusicaux de son époque ${ }^{3}$. Bien qu'il soit souvent difficile de cerner à quel point les propos polémiques de Margat sont les siens ou constituent eux-mêmes des caricatures ironiques (est-ce le conservatisme qu'il oppose aux changements de la modernité sincère ou s'agit-il plutôt d'une raillerie des bienpensants ? ${ }^{4}$ ), il n'en demeure pas moins que ses billets promeuvent une vision idéaliste de la musique considérée comme le produit intouchable ${ }^{5}$ de $1^{\text {'inspiration du }}$ génie. Le discours esthétique se cache donc aussi dans l'humour et peut même en être la victime.

D'où le titre du présent article, qui reprend la forme des titres de Margat, dans lesquels s'ajoute parfois ${ }^{6}$ à la partie fixe "Variations... sans thème " une désignation expressive en italien - comme dans une partition - écrite en italique entre parenthèses, qui se veut une caractérisation du billet: on y retrouve des expressions classiques (quasi fantasia, mezza voce, con grazia, scherzando, poco meno mosso, attacca subito l'allegro, etc.), des variations sur celles-ci (feroce e gajamente, poco malinconico, serioso ma non troppo, etc.), des formules qui peuvent rappeler l'esprit d'Erik Satie (D. C. ma senza replica, innocente, senza rigore, quasi tremolo, etc.) ; il s'agit parfois de pseudo-italianismes mal orthographiés (risolato pour " risoluto ", harmonioso pour " armonioso ", simplice pour "semplice», etc.). Nous allons donc explorer l'humour estetico des "Variations... sans thème » en proposant un parcours enrichi d'extraits de ces billets (en gris, selon la convention de $1^{\prime}$ « Anthologie du PHEM » ${ }^{7}$ ).

1 Le Guide du concert reprendra sa publication en novembre 1945. Une fiche descriptive de la revue est disponible ici : http://pressemusicale.oicrm.org/corpus/periodiques/revues-musicales (toutes les pages internet citées ont été consultées pour la dernière fois le 11 avril 2018).

2 Voir à ce propos notre texte de présentation du projet « Histoire de l'esthétique musicale en France, 1900-1950» (PHEM) (Lazzaro 2017).

3 Une fiche bio-bibliographique sur Margat, compositeur et professeur de musique privé, est consultable ici : http://pressemusicale.oicrm.org/corpus/musicographes/. Les références aux "Variations... sans thème " dans le texte et dans les notes suivent le modèle "VsT X/Y ", où X correspond au volume du Guide du concert et $\mathrm{Y}$ au numéro de fascicule où le billet est publié.

4 Dans le billet du 9 décembre 1938 ( $\underline{\text { VST 25/10 }}$ ), Margat critique explicitement les réactionnaires, " ces derniers tenants de la légalité et de l'ordre, dont [il] respecte les convictions et dont, sur beaucoup de points, [il] partage d'ailleurs le sentiment ». Margat leur reproche que "l'idée qu'ils se font des procédés de la technique moderne est peut-être un peu sommaire ", en d'autres mots, il ne cautionne pas l'attitude méprisante de ceux qui disent que " pour composer, de nos jours, il suffit de poser, à plat, ses deux mains sur le clavier et d'écrire, en pagaille, sur deux portées, ce qui en résulte ».

5 Voir les critiques de Margat aux arrangements : VsT 24/20, 25/28-29.

6 Plus précisément, cette sorte de sous-titre se retrouve systématiquement durant la première année (1934-1935) de la série, et disparaît ensuite.

$7 \quad$ Nous avons présenté cette série dans le vol. 4, n 2 de la Revue musicale OICRM (Lazzaro 2017). Note sur les transcriptions des textes : dans la transcription des textes, nous adaptons tacitement la ponctuation, l'usage des majuscules, des italiques et des guillemets aux normes typographiques modernes. 
À travers les propos de Margat, il s'agira d'explorer tout d'abord la crise (sociale et musicale) qu'il dénonce, pour ensuite présenter les solutions (nostalgiques et pédagogiques) qu'il propose, tout en portant une attention particulière à la façon dont le style ironique de l'écriture véhicule des prises de position esthétiques. L'analyse des "Variations... sans thème ", en plus d'être intéressante en raison de l'ampleur de cette rubrique (126 billets en total), est essentielle pour étudier la place et les formes du discours esthétique et social sur la musique dans $L e$ Guide du concert. On constate en effet que la naissance de la rubrique de Margat au début du volume 21 de la revue coïncide avec une diminution drastique de la publication d'articles à contenu esthétique : durant les quatre ans et demi compris entre octobre 1934 et l'arrêt de la revue en mai 1939, mis à part une série d'articles sur le drame wagnérien (Ferchault 1938), un article sur le « jazz hot » ([Nourry $]$ 1937) et un autre sur « La musique allemande après Hindemith " (Barraud 1938), les «Variations... sans thème » restent le seul endroit où Le Guide du concert continue d'offrir à ses lecteurs un contenu autre que le calendrier des concerts (en salle et radiophoniques), l'analyse des œuvres au programme, des entretiens ou des portraits historiques. Même une enquête au sujet potentiellement très propice au débat comme « Où va la musique? " ([Anonyme] 1936), proposée par la rédaction au début du volume 23 (octobre 1936), ne donnera lieu à aucune réponse publiée (sauf une "Variation » de Margat qui peut être considérée comme une réplique à l'enquête ${ }^{8}$ ) - chose étonnante si l'on pense aux réactions nombreuses et souvent très riches suscitées par les enquêtes lancées par le Guide dans les années 1920 et au début des années $1930^{9}$. Margat assume donc un rôle éminent auprès des lecteurs du Guide du concert, grâce à sa présence constante et à l'exclusivité des thèmes dont il traite. Ses billets offrent un point d'accès aux sujets de discussion du milieu des amateurs de musique parisiens de la seconde moitié des années 1930 ; ils les reflètent et les encouragent, et en partagent probablement le ton d'humour polémiste placidement conservateur. Margat tisse l'éloge de ces dilettantes qui ne sont pas pour autant des "bricoleur[s] maladroit[s] » ou des « touche-à-tout prétentieux », mais qui n'ont nul autre but que "le plaisir des siens et [leur] propre agrément " (VsT 21/7). Ce public de dilettantes doués, lecteurs du Guide du concert, constitue d'ailleurs la cible des cours d'introduction à l'harmonie que Margat donne depuis novembre 1932 et qui sont amplement publicisés par la revue sous forme d'annonces, d'interviews ou de lettres ouvertes accompagnées de l'effigie du professeur en couverture (figures 1 à 4), ce qui contribue à investir le musicographe d'une autorité professorale donnant du crédit à ses écrits.

La graphie originale des noms est respectée. Toute autre intervention est signalée en note. Les coupures sont indiquées par « [...] » ou bien remplacées par un résumé de leur contenu présenté en italique et entre crochets.

$8 \quad$ VST 23/3. Voir plus bas dans la section "Musique de la crise ». Une série de deux articles intitulés "Où va la musique ? " paraîtront quelques mois plus tard dans Le Ménestrel, signés par Raoul Brunel (pseudonyme de Raoul Blondel ; voir sa fiche biographique ici : http://pressemusicale.oicrm.org/corpus/ musicographes). Le lien de cette prise de parole avec l'enquête lancée par le Guide n'est toutefois pas explicité (Brunel 1937).

$9 \quad$ La musique et le sport, 1924-1925 : 79 réponses publiées ; La musique immorale, 1925 : 43 réponses ; La musique mécanique, $1928: 35$ réponses ; Audition directe et audition indirecte, $1930: 26$ réponses ; Les applaudissements au concert, $1932: 32$ réponses. 
Figure 1. Portrait photographique d'Yves Margat par Pierre Auradon sur la couverture du Guide du concert, vol. 24, $n^{\circ} 4$ (22 octobre 1937).

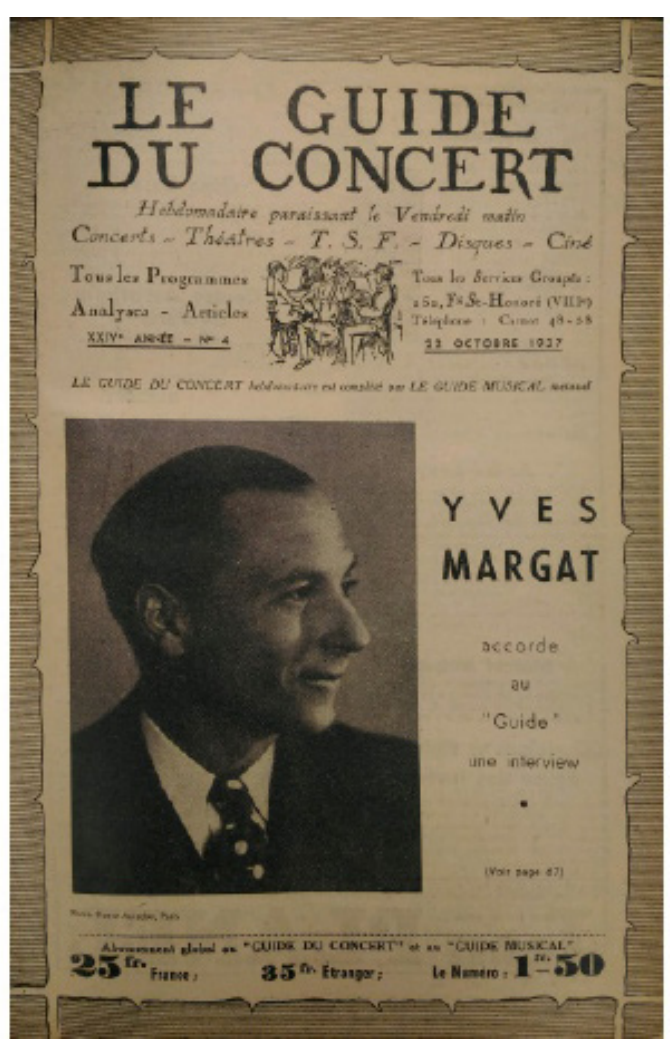

M. Yves Margat a repris ses cours et leçons particulières d'harmonie, 9, avenue Victor-Hugo $\left(16^{\circ}\right)$.

Il doniera, au cours de la saison Chopin, un concert entièrement salson, Salle premières ouvres de ses élèves : musique instrumentale, mélodies, plano, orchestre.

Cette anré, t:*

Cette anné, comme les précécientes, $M$. Cours préparatoir à partir de novembre, un tants (amateurse gratuit réservé aux débufet duquel toutes et professionnels) eiv au sul'horaire nées crns le mode d'inscription seront don-

En marre $* *$

bituel, le de son docte enseignement hacrera, le jeune et distingué professeur consavité à cette année, une partie de son actiet le chantmation, par le piano, le solfége ces musint conjugués, des jeunes intelligen7 ans pourron. Les enfants, à partir de 6 et de choix précoce et seront appeles à faire valoir leur teur au talent de pianisie et d'accompagni temps, Pours d'une audition dès ce prinvous, telur tous renseignements et rendezPassy 28-56.
Figure 2. Annonce des cours d'Yves Margat dans Le Guide du concert, vol. 23, $n^{\circ} 1-2$ (2 et 9 octobre 1936), p. 2. 


\section{LIE CUUDE WU CONCERT \\ Directour : Gabtiel BEIDER \\ Tusphone : ligne perticullere carnot 40-58 \\ Fixiception : JEUDI \& 8 E heares \\ 5tendard Carsat oe.so et suite \\ hatros bouts aur rendarewors}

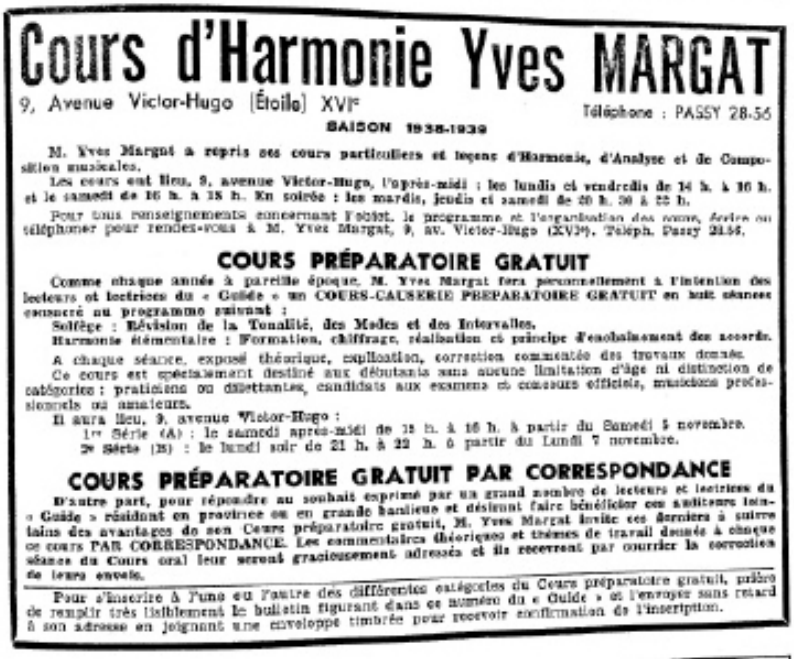

Figure 3. Descriptif des cours d'Yves Margat (enrichis du nouveau cours par correspondance) sur le frontispice du Guide du concert, vol. 25, no 4 (28 octobre 1938), p. 69.

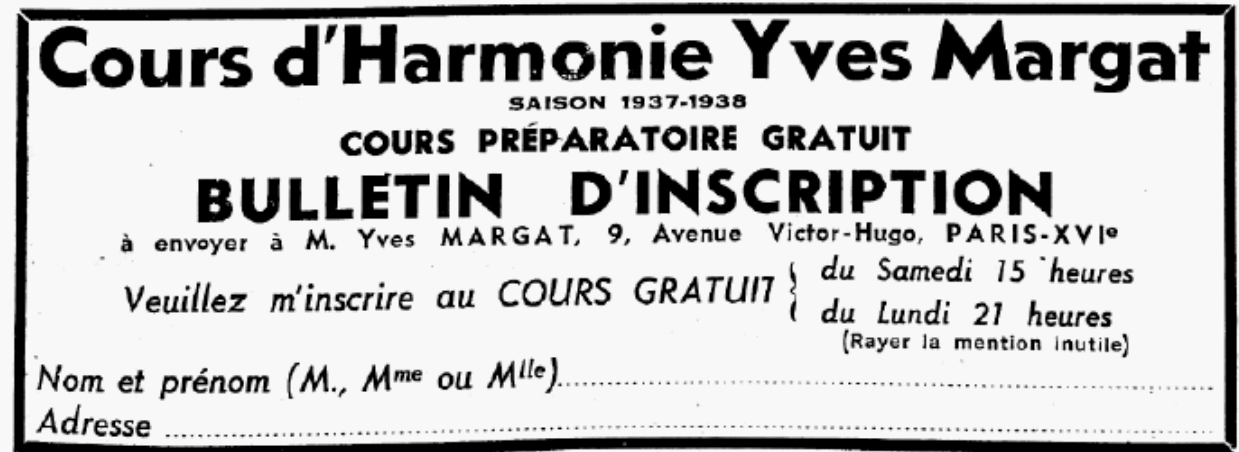

Figure 4. Bulletin d'inscription au cours gratuit pour les lecteurs $d u$ Guide du concert sur le frontispice du vol. 24, no 5 (29 octobre 1937), p. 101.

MUSIQUE ET CRISE

Le premier billet de la série ("Variations... sans thème (con bravura) ", VsT 21/3) donne le ton : en périodes de crise, la musique « devient un besoin et un refuge ». Cet objectif programmatique est le la que Margat donne à sa cigale métaphorique pour qu'elle chante :

La cigale ayant... dormi tout l'été s'éveille, l'heure d'hiver venue. Elle ouvre un volet, puis deux et met le nez à sa fenêtre.

- Hum ! Ce pâle matin d'octobre ne m'inspire rien qui vaille. La bise déjà ! de vilains nuages à 1 'horizon... Brrr ! le joli temps pour ma rentrée ! 
La cigale a le réveil chagrin. Pour sûr, tout n'est pas rose dans le paysage. On le sait, parbleu, et il ne manque pas de gens pour nous le rappeler. La politique ! la révolution !! la guerre !!! la crise !!!! Autant de perspectives réfrigérantes. [...]

Dame cigale, ma mie, vous avez une mission à remplir céans. En période de prospérité, quand tout va trop bien, la musique n'est souvent qu'un luxe ou un aimable délassement. Par ces temps de détresse universelle, quand rien ne va plus, elle devient un besoin et un refuge. Allons, cigale, à l'ouvrage. Voici le la.

Chantez, chantez ma belle...

Nous sommes en octobre 1934, en pleine période de crise économique (qui, en France, se situe entre 1931 et 1935) et politique (particulièrement vive depuis les émeutes du 6 février), avec le gouvernement de trêve de Gaston Doumergue (droite) engagé dans une réforme de l'État qui n'aura pas lieu et qui mènera plutôt à une crise du gouvernement, remplacé en novembre par les radicaux de Pierre-Étienne Flandin ${ }^{10}$. Entre temps, on assiste à la création du Front populaire (dont un des moments constitutifs remonte au pacte signé entre socialistes et communistes le 27 juillet 1934), qui prendra le pouvoir en avril 1936 pour le garder, de manière de plus en plus difficile à partir de l'été 1937, jusqu'en 1938 (avril : fin du second gouvernement de Léon Blum ; octobre : dissolution du Front populaire à l'issue du congrès radical de Marseille).

Plusieurs billets de Margat sont des commentaires indirects, souvent transposés - ironiquement ou polémiquement - au monde de la musique, des effets de la crise sur la population ainsi que des revendications, des nouvelles lois et des réformes sociales transformant la vie des Français (notamment les conquêtes du gouvernement de Léon Blum que sont la semaine de travail de 40 heures et les congés payés). Par exemple, dans un billet ironique de fin 1936, Margat transpose les luttes syndicales "sans précédent » qui ont caractérisé l'été $1936^{11}$ au monde de l'enseignement musical : il imagine une grève des professeurs de piano contre la « CPPPPPP (Chambre Patronale des Parents Pratiques qui Préfèrent le Phono au Piano) » en appui de leurs revendications d'une semaine de travail de minimum 40 heures et de "l'enseignement de plus de trois instruments distincts, comme, par exemple, le bugle, la guitare hawaïenne et le piano déclaré incompatible et illégal " ( $\left.V_{s} T 23 / 3\right)$. Ce billet pourrait être considéré comme une réponse à l'enquête «Où va la musique ? » lancée par Le Guide du concert dans le numéro précédent, qui incitait ses lecteurs à

examiner objectivement, en dehors de toute théorie et de toute opinion politique, les effets qui vont avoir les récentes lois sociales (congés payés, relèvement des salaires, semaine de 40 heures, dévaluation du franc) sur les diverses activités de la musique, afin de déterminer les mesures qu'il y a lieu de prendre pour essayer d'adapter la vie musicale à la nouvelle évolution sociale ([Anonyme] 1936).

10 Pour un portrait synthétique de la crise économique et politique en France, voir par exemple Berstein 1988, chap. 2 et 3 ou Sirinelli 1993, chap. 4.

11 Sur ce « mouvement de grève sans précédent », voir entre autres Vigreux 2016, chap. 4. 
La crise de l'enseignement musical est d'ailleurs un thème récurrent chez Margat, qu'il s'agisse de dénoncer « l'exercice illégitime du professorat musical » qui plonge le corps enseignant dans de " graves difficultés matérielles et morales » (VsT 21/4); de constater qu'uniquement un cinquième des professeurs de musique français peuvent se vanter d'une " autorité professionnelle indiscutée " dérivant d'une affiliation officielle (ce qui mène à une "génération spontanée de titres flatteurs autant qu'obscurs dont s'orne, sans remord [sic], tout bristol qui se respecte » (VsT 24/4)) ; d'inviter les parents d'élèves à payer convenablement, préférablement en nourriture vu la dévaluation du franc, le professeur (qui « n'a pas besoin d'argent. Il vit de bonne soupe... » (VsT 25/5)), contraint à donner ses cours à domicile pour arriver à la fin du mois (VsT 21/21); de regretter le temps où il était encore possible, pour un jeune diplômé, d'organiser son premier récital (qui se donne désormais "débout... dans les cours, avec la complicité attendrie des mesdames les Concierges » (VsT 22/15)) ; de tisser l'éloge des emplois musicaux de série B, comme celui de tourneur de pages (" tout indiqué " pour un " musicien momentanément sans emploi et qui cherche une occasion de se reproduire »(VsT 24/18)). Si les temps sont durs pour les professeurs de musique, selon Margat, c'est en raison du manque de considération générale pour la pratique musicale due à diffusion de la radio et des disques (nous y reviendrons) et à la concurrence des loisirs sportifs. Plus personne ne fait de la musique, et l'État préfère investir ailleurs. Dans un billet aux allures dystopiques qui imagine, au contraire, que la pratique musicale devienne une véritable concurrente de la pratique sportive durant les nouveaux congés octroyés aux travailleurs ${ }^{12}$, Margat appelle à des mesures d'urgence " si l'on veut circonscrire le fléau et endiguer cette vague de musiciens et musiciennes à tous crins et de tous calibres qui menace de déferler sur le territoire » :

Recensement et réquisition immédiats des clairons, trompettes, cloches et autres engins sonores présentement détenus par les particuliers, en vue d'une redistribution ultérieure par les soins des communes, après examen d'un médecin aliéniste et avis favorable d'un professeur du Conservatoire ; création de vastes musicodromes à distance suffisante des agglomérations et où les masses mélomanes pourront se livrer à leur sport favori ; construction de kiosques souterrains ; réglementation de la vente des instruments à percussion ; arbitrage obligatoire par la concierge des conflits pouvant surgir, l'été, entre locataires-instrumentistes du même immeuble, etc.

A-t-on prévu, en outre, la situation difficile des professeurs de musique, qui, du jour au lendemain, vont se trouver débordés? (VsT 23/31-32).

Le désintérêt des masses pour la Musique avec un grand " $\mathrm{M}$ " et la crise économique engendrent une tristesse diffuse chez les musiciens. Margat remarque à plusieurs reprises, à partir de 1934 et avec plus d'insistance en 1937-1938 (VsT24/1-2, 24/7, 24/28-29-30, 25/25-26-27), que "la vie n'est drôle pour personne » et que " les musiciens ont habituellement l'air triste » (VsT 21/8), même dans leur façon

12 «Pour occuper les loisirs abondants que le législateur vient de lui octroyer, le Français qui ne fréquente pas les stades, ne joue pas la belote, ne monte pas à tandem, n'a pas de goût pour la peinture, ignore les joies captivantes de la pêche à la ligne, des échecs et des mots croisés, n'a pas de petits pois à ramer et ne sait pas nager... n'aura d'autre ressource que de sombrer dans la musique » (VsT 23/31-32). 
de s'habiller (VsT 23/16). Il condamne l'inutilité du diplôme du Conservatoire : "Dans l'ère d'universelle dévaluation que nous vivons, le titre de Premier Prix n'est comme tant d'autres qu'un titre-papier et le diplôme, même dans son cadre, est loin aujourd'hui de valoir son pesant d'or...»(VsT 23/9). Ces lamentations sont sans doute une manifestation de l'amertume du lauréat Margat envers ses collègues qui ont réussi dans leur carrière. On le comprend dans ses attaques contre les virtuoses gâtés par le public (VsT 22/9) ; on soupçonne un alter ego lorsqu'il parle d'un ami compositeur « méconnu, mais non sans talent » (ibid.); on saisit sa peine lorsqu'il narre l'occasion perdue de devenir chef d'une fanfare improvisée pendant la guerre : "Voilà comment, une fois dans ma vie, j'ai failli devenir chef d'orchestre. Je ne retrouverai jamais une occasion pareille... " (VsT 22/21)). Avec un paternalisme cavalier qui était loin d'être exceptionnel à l'époque, Margat salue en revanche l'un des effets à son sens positifs de la période de crise : le nombre de plus en plus élevé de femmes s'orientant vers la musique professionnelle ${ }^{13}$ :

Si l'agriculture manque de bras masculins, la main-d'œuvre féminine n'est pas près de faire défaut dans cette branche secondaire de l'activité nationale qu'est la musique. Conséquence imprévue de la crise ? Ou signe des temps ?... La femme s'oriente de plus en plus vers l'art où s'illustra Chaminade ${ }^{14}$. [...] Moins par galanterie que par indifférence, [le sexe fort] s'efface pour La laisser passer et Lui abandonne sans combat une carrière qui, par le temps qui court, ne nourrit plus son homme.

De toutes les conquêtes du féminisme, celle-là me semble la moins inquiétante et la plus indiquée. Le métier de musicien est encore, dans le brutal struggle for life moderne, un des plus décents qu'une jeune fille puisse envisager, un de ceux qui répondent le mieux à son instinct d'élégance, à son goût du sérieux et à ses aptitudes intellectuelles et sentimentales. Physiques aussi... Il est des adaptations féminines moins heureuses.

Ne serait-ce que du seul point de vue esthétique - point de vue qui peut avoir son agrément - l'image d'une aimable " moins de vingt printemps " maniant avec grâce l'archet ou laissant courir de jolis doigts sur le clavier me séduit infiniment plus, je l'avoue, que le petit short crotté d'une rugby-woman roulant dans la boue ou que la grimace très sportive de la championne des 100 mètres à l'arrivée, soufflant comme une Pacific... au départ ${ }^{15}$ (VsT 21/19).

13 Deux ans après, par contre, en constatant l'inutilité des études en musique pour se nourrir, il n'imagine pour la femme musicienne qu'un rôle d'épouse et mère qui pourra apprendre les gammes à ses enfants (VsT 23/9).

14 Cécile Chaminade (1857-1944), pianiste et compositrice, première musicienne à être promue Chevalier de la Légion d'honneur en 1913, la même année que Lili Boulanger, remportait, première femme, le premier Grand Prix de Rome en composition.

15 Margat fait ici référence au modèle de locomotive par ailleurs chanté par Arthur Honegger dans son « mouvement symphonique » Pacific 231 (1923). Le thème de la musicienne attirant les regards lubriques revient chez Margat à quelques occasions. Par exemple : " la haute composition musicale s'érigeant de plus en plus en sport féminin, il advient que les "belles écouteuses" du balcon aient la déception de voir s'installer au piano, en guise de queue-de-pie, un Cher Maître en robe du soir, dont l'irréelle blondeur et le décolleté... bien réel, lui, s'intellectualisent d'une inutile paire de lunettes d'écaille. C'est alors au tour de Messieurs les mélo... mâles de l'orchestre de ne plus écouter avec toute l'objectivité désirable » (VsT 24/17). 
La musique que Margat chérit est l'expression de la délicatesse opposée à la brutalité moderne incarnée par les sports et les machines (dont l'esprit rentre dans le domaine musical, conquis par la chasse aux records : "le plus grand pianiste in the world sera le plus "vite" " (VST 25/32-33)). La pratique musicale est conçue comme une évasion du monde en crise, comme un " désintoxiquant » (Margat dans P. V. 1936). Comme il l'explique dans une (fausse ?) interview publicisant ses cours d'harmonie,

l'essentiel est de pouvoir, de temps à autre, fuir l'obsession des soucis quotidiens et des préoccupations bêtement terre-à-terre en passant, égoïstiquement [sic], une heure avec soi-même, dans l'isolement d'un divertissement intellectuel qui soit une évasion, penché sur une tâche à laquelle nul ne nous oblige et qui soit un délassement. La musique peut être cette distraction choisie. L'harmonie, grâce à l'attrait de ses petits problèmes stratégiques d'écriture, à la diversité de ses combinaisons, à la perfection de sa logique, aux résonnances qu'elle éveille peut devenir cette tâche captivante attendue secrètement tout le jour et remplie avec délectation, le soir venu (X. 1937).

Les bienfaits d'un "violon d'Ingres " reviennent souvent dans ses écrits et sont développés dans le billet suivant (VsT 23/5) :

Quand il avait assez de peindre, Monsieur Ingres saisissait son violon et se régalait d'un peu de musique. [...] Vivant d'un beau métier et en des temps plus glorieux, hélas ! que les nôtres, Ingres n'en éprouvait pas moins ce besoin d'évasion qui est au fond de chacun de nous, prisonniers que nous sommes entre les quatre murs où nous enferme la routine quotidienne de nos travaux, de nos soucis et de nos loisirs. Et c'est à la musique qu'il demandait cet « autre chose » après quoi nous aspirons tous confusément.

La musique! Route directe des grandes évasions, invitation au plus beau des voyages vers les paysages dont on rêvait et qui ne nous décevront pas, délivrance et apaisement de l'esprit, source claire où l'âme se mire et ne se reconnaît plus, tant elle se découvre de jeunesse, d'enthousiasme et de foi... Quel merveilleux violon d'Ingres !

Puisque la musique ne doit pas être contaminée par l'impératif de l'efficacité, Margat s'exprime à plusieurs reprises contre sa marchandisation, et plus particulièrement contre les contacts entre la musique et la publicité - dans les deux formes (a) de musique au service de la publicité (jingles radiophoniques) et (b) de la publicité servant à financer les activités musicales :

(a) Depuis la fin des mirlitons, il ne s'est point fait une aussi furieuse dépense de quatrains et de distiques, échantillons non sans valeur de poésie lapidaire, où l'opulence de la rime compense l'objectivité parfois un peu prosaïque du propos. Quant aux romances et rondes d'autrefois, ariettes qu'on croyait oubliées, c'est plaisir de le réentendre, toujours jeunes et aussi charmantes, vous affirmer avec l'accent de la sincérité la plus désintéressée et le trémolo le plus persuasif la supériorité imbattable d'un café ou d'une pâte alimentaire, la longévité d'un mobilier - il faut le casser à coups de marteau - ou, surtout à l'heure du déjeuner, l'action souveraine, indolore et immédiate de certaine tisane contre la paresse intestinale (VsT 22/33-34). 
(b) Entendrons-nous, bientôt, avant l'attaque du $1^{\mathrm{er}}$ mouvement du Concerto en ré dièse mineur de X..., le chef d'orchestre déclarer : "Ce concert vous est offert par la pâte à rasoir Y... ». Ou, mieux encore, sur le plateau de l'Opéra, le châtiment final du Traître étant intervenu dans les formes et délais habituels et la bienveillante inattention générale, verrons-nous le régisseur de la scène venir réclamer une minute de silence pour informer les spectateurs que le drame lyrique en 5 actes et 14 tableaux auquel ils viennent d'assister leur a été offert par la tisane Z..., le plus soporatif des somnifères ? Suivrait l'inévitable petit distique publicitaire, repris par les chœurs au grand complet et fredonné par le public tout le long du grand escalier (VsT 23/4).

En donnant à la musique la fonction d'évasion du quotidien, Margat lui nie toute fin utilitaire, ne serait-ce que pour le bien-être du corps - une " musicothérapie " contre laquelle Margat écrit un de ses billets le plus sarcastiques ${ }^{16}$. Toujours dans cette lignée, il décrie les opéras à sujet réaliste (a) et les mises en scène réalistes des opéras traditionnels (b) :

a) Si la musique est la langue de l'ineffable, pourquoi s'en servir là où les mots ordinaires suffiraient? Il y a la prose, il y a les vers, puis il y a la Musique. [...] L'ancienne formule de l'opéra-comique était dans le vrai. [...] Mais qu'il faille vingt-cinq pages d'imprécations lyriques et treize cents notes, dont sept « contre-ut » pour nous raconter une de ces histoires de famille comme on en lit tous les jours, en trois lignes, dans son journal ! (

b) Le théâtre - et surtout le théâtre lyrique - n'est pas la vie courante. Nous y venons précisément chercher tout le contraire. Les anomalies de la mise en scène, l'invraisemblance des péripéties, le convenu des situations et des attitudes nous reposent de l'inflexible logique habituelle et du réalisme quotidien ( $\underline{\operatorname{Vs} T 23 / 23})$.

Dans une période de grande politisation de la vie sociale, Margat s'exprime à propos de la politisation de la musique. Si les compositeurs ont le droit d'avoir des opinions politiques, il est convaincu que celles-ci ne devraient pas se confondre avec le domaine artistique. Celle qu'on appelle "l'extrême-gauche musicale ", étiquette courante (avec «bolchévisme musical») dans la presse pour désigner l'avant-garde non tonale ${ }^{17}$, n'est pas pour autant - met en garde Margat - liée à la gauche politique ${ }^{18}$ :

\footnotetext{
16 «Nous trouvions déjà, en la Musique, un remède souverain contre les tourments de l'âme. Nous ne nous serions pas avisés d'avoir recours à elle pour le soulagement de nos infortunes physiques. Après l'hydro..., l'électro... et la sympathicothérapie, la musicothérapie. Pourquoi pas ? La musique vient bien à bout de l'insomnie la plus opiniâtre ! [...] Au lieu d'aller aux Eaux, on fera chez, en famille, sa petite cure de musique de chambre. À moins que le docteur ne vous prescrive une saison à Bayreuth ou à Salzbourg érigées en stations thermales. On prendra le soir, avent de s'aller coucher, deux comprimés de Chopin ou une bonne infusion de Mendelssohn. [...] Médecin malgré lui, le professeur de musique paiera patente. Les leçons - au cachet - deviendront des consultations ; le Conservatoire, une annexe de l'École de Médecine, et le trombone à coulisse, un instrument chirurgical »(VsT 24/12-13-14).

17 Voir à ce sujet Lazzaro (sous presse), chap. 7.

18 Sur les politiques musicales du Front populaire et sur la question du style politically correct, voir Moore 2006 ; 2008.
} 
Nous avons bien, je sais, une musique dite d'avant-garde et des compositeurs d'extrême-gauche que certaine politique partisane, se méprenant sur l'étiquette, s'est mise en devoir de revendiquer. Comme si les audaces contrapuntiques, l'agressivité polytonale ou le sectarisme instrumental vous enrôlait d'office un homme - au demeurant le plus bourgeois et le plus inoffensif du monde - dans la croisade de l'anti-fascisme ! À ce compte-là, celui qui se hasarderait à résoudre une septième serait aussitôt considéré comme suspect et fourré en prison en qualité de factieux. Car nous avons, aussi, une production de centredroit, un soupçon conservatrice, et entre ces deux extrêmes, pas mal de modérés, indécis et opportunistes de toute nuance. Voyez où cela nous conduirait si la plus innocente Sonate devait être interprétée comme une adhésion au programme du Rassemblement populaire ou comme l'aveu d'une infâme collusion avec la réaction... selon les préférences harmoniques de son auteur. Heureusement, nous n'en sommes pas là. Tout ce que demandent présentement les musiciens - d'accord en cela avec un certain nombre de Français moyens - c'est que la politique ne les empêche pas de continuer à cultiver en paix leur jardin et d'y faire fleurir... de la musique avant toute $\operatorname{chose}^{19}$ (VsT 23/10).

\section{CRISE DE LA MUSIQUE}

Quelle musique invoque Margat? Sous quelle forme veut-ill'entendre ? Le billettiste est un partisan de la musique d'art consacrée par la tradition, tout en étant ouvert à la redécouverte de partitions moins jouées (comme les sonates de Mozart, qu'il invite à reconsidérer comme des chefs-d'œuvre et non seulement comme des exercices pour jeunes pianistes (VsT 22/17)), et en promouvant la curiosité pour toute musique bien écrite. En fin connaisseur de l'écriture, il s'intéresse à la musique "digne de ce nom » : "Seuls le mauvais goût, la platitude et la malfaçon devraient motiver son indifférence ou son mépris » $(\underline{V s T} 25 / 21)^{20}$. Ainsi il incite ses lecteurs, pour être de "véritable[s] amateur[s] ", à ne pas s'arrêter à leur auteur préféré mais à " élargi[r] le cercle de [leurs] relations musicales » (ibid.).

Il se met du côté de ceux et celles qui aiment les airs les plus célèbres du Faust de Gounod en défendant celles-ci contre les accusations d'être des « rengaines " banales (" quel est le compositeur qui, dans le secret de son cœur, ne souhaiterait en avoir réussi... une ou deux pareilles ?» $(V s T 21 / 11))^{21}$. Vous ne devez pas avoir honte d'aimer les mélodies faciles à retenir, Margat rassure ses lecteurs, vous ne devez pas forcément connaître tous les détails d'une partition pour pouvoir apprécier une œuvre à sa juste valeur. Cependant, accuse sarcastiquement Margat, le secret du succès de la musique contemporaine est qu'elle soit impossible à retenir : « La pire disgrâce qui puisse arriver aujourd'hui à une mélodie est d'être "demain" sur toutes les lèvres ", et les compositeurs doivent espérer, pour leur gloire, que leur œuvre soit " sifflée dans la salle plutôt que sifflotée dans l'escalier» ( $\underline{\text { V T 23/19 }}$; voir aussi VsT 25/34). En dépit de cet amer constat, Margat incite les compositeurs à retrouver la "gaieté franche,

19 Célèbre premier vers de l'« Art poétique » de Paul Verlaine (dans Jadis et naguère, 1884).

20 Margat est contre la musique vulgaire sauf si elle est une «tradition », comme le Cantique de Noël d'Adolphe Adam (VsT 22/12-12-14).

21 Sur le succès perdurant de Faust, voir aussi VsT 21/16. 
saine, spontanée, déboutonnée un peu sinon débraillée [...], bonne fille, complaisante si l'on veut, mais gaieté... de cœur et sans fard » (VsT 24/7), une gaieté qu'il considère très française et qui a été abandonnée, d'une part, en faveur d'une approche ironique pince-sans-rire (" gaieté froide trop ingénieusement calculée qui s'extériorise au moyen de cocasseries et de parodies instrumentales ou de loufoqueries harmoniques et rythmiques perpétrées avec préméditation ", ibid.), et, d'autre part, en faveur de partitions « sévères, laborieuses et... décourageantes » (ibid.) qui souvent suscitent une réaction comique involontaire (VsT 24/1-2) 22 .

Dans le cadre du hiatus existant entre compositeurs modernes et public, Margat se place parmi "l'immense foule des auditeurs de bonne volonté ", ces amateurs sincères qui ne comprennent pas la musique contemporaine, et qui, à la différence des pharisiens constituant « 1'Élite, [les] Initiés, [1']Avant-Garde » et de « l'arrière-garde », " n'ont pas honte de l'avouer... eux!" (VsT 21/10). Margat connaît bien ses lecteurs, il sait qu'ils sont en grande partie ces auditeurs dépaysés auxquels il offre, avec autorité, son appui. Il comprend leur détresse et les encourage, tout en caractérisant ironiquement les autres. À travers sa dérision de l'adversaire, Margat crée des adeptes de son crédo esthétique.

"S'il est inévitable qu'une langue évolue, il est désirable qu'elle n'évolue pas trop vite ». Cette sage réflexion d'un grammairien contemporain peut s'appliquer, ce me semble, au langage musical. Les mêmes raisons qui s'opposeraient à un bouleversement subit des règles et des traditions du langage parlé militent contre les tendances révolutionnaires d'un petit nombre de Jeunes Turcs ${ }^{23}$ aux dents pointues et à l'oreille dure qui prétendent réviser, à leur façon, la Constitution musicale...

Que feraient les derniers habitués du Théâtre-Français si, en manière d'innovation, on se mettait, un beau soir, à leur servir une prose où les mots n'occuperaient plus la place que leur assigne l'usage et où la désinence des participes et l'accord de verbe seraient soumis aux caprices de la seule Fantaisie?

Toute proportion gardée, c'est un peu ce qu'on voit en musique.

Seulement, pour avoir raté, naguère, le train de Carmen ou celui de Pelléas ${ }^{24}$, aujourd'hui tout le monde veut être du dernier bateau, sans s'inquiéter du vent qui les pousse, fût-ce celui de la Folie...

Quand je dis « tout le monde », je pense à l'Élite, aux Initiés... à l'Avant-Garde. Car il reste l'immense foule des auditeurs de bonne volonté qui composent le gros de l'armée. Ceux-là marchent encore... mais il y a beau temps qu'ils ne comprennent plus, et, ma foi, ils n'ont pas honte de l'avouer... eux!

22 Sur le thème de la musique gaie, voir aussi $V_{s} T$ 25/25-26-27.

23 La référence est à l'aile gauche du Parti radical, dont les militants (Albert Bayet, Gaston Bergery, Pierre Cot, Bertrand de Jouvenel, Jacques Kayser, Jean Mistler, Pierre Mendès France, Gaston Riou, Georges Potur, Jean Zay, etc.) prônaient pour une réforme draconienne de l'État dans l'esprit des Jeunes-Turcs ayant renversé le sultan Abdülhamid II en 1909. Voir Berstein 1982 ; 1988, p. 87 ; 2003. L'étiquette « Jeunes Turcs » était d'ailleurs plus largement utilisée, dans l'entre-deux-guerres, pour désigner les mouvements contestataires ; voir Cochet 2008.

24 Carmen de Georges Bizet et Pelléas et Mélisande de Claude Debussy sont deux opéras partageant la réaction choquée qu'elles ont provoquée lors de leur première (respectivement en 1875 et 1902) et la transformation, par après, du choc en monumentalisation. 
Et je ne parle pas de l'arrière-garde qui, loin sur la route, trottine sans l'espoir de rattraper jamais le peloton de tête... (VsT 21/10).

L'adversaire ridiculisé par Margat n'est jamais précisé. Ses «Variations » sont, ainsi, véritablement "sans thème ", en ceci qu'elles ne sont pas des critiques d'un concert, d'une œuvre ou d'un artiste en particulier. Il y a plutôt des catégories récurrentes, notamment les artistes médiocres et les jeunes compositeurs (les Jeunes Turcs du passage que nous venons de citer) avec tous leurs défauts : le caractère vide de leur musique (vide d'inspiration, vide d'émotion, vide de contenu musical ${ }^{25}$ ) et leur apparent masochisme envers les oreilles du public ${ }^{26}$. Comme corollaire, Margat s'en prend à l'accueil trop enthousiaste et immérité de cette musique (ce qu'Esteban Buch appelle « le complexe de Hanslick » (2006, p. 79) : "Dans la crainte de laisser passer encore une fois le Génie sans le reconnaître, on en découvrirait plutôt dans la première loufoquerie venue, fût-ce une Rapsodie pour deux timbales et basson sur des thèmes de l'Ouganda... " (VsT 24/23). En effet, une des caractéristiques de la " cuisine futuriste " - que Margat suggère de renfermer dans des pots de conserve à " ne point consommer avant l'an 2000 » (ibid.) - est d'être internationale, ce à quoi Margat fait constamment des clins d'œil, des déjà cités " thèmes de l'Ouganda " au "final de certain Concerto letton-slovaque »(VsT 24/1-2), en passant par la pléthore de noms étrangers qui occupent les scènes parisiennes. Margat est-il un porte-parole de l'esthétique nationaliste clamant la supériorité de la musique française ? Malgré l'évidence d'un certain mépris pour le cosmopolitisme parisien, sa crainte est que les artistes français se dotent d'un pseudonyme exotique pour rivaliser avec les « vedettes cosmopolites » et que ce "nom de rechange » cache en réalité une œuvre médiocre (l'artiste médiocre, voilà donc une autre catégorie souvent soumise aux attaques de Margat) :

Défense est faite aux artistes allemands d'emprunter dorénavant un pseudonyme à désinence étrangère... Ce décret vient d'être pris outre-Rhin. Cela ne m'empêche pas d'en approuver - sinon la manière - du moins l'esprit. Toute xénophobie de côté, je ne vois guère, pour un musicien français, par exemple, la nécessité d'un nom de rechange, qu'il sonne en am, en ski ou en... toc.

$[\ldots]$

25 Voir VsT 24/8, où Margat critique l'attention exagérée portée à l'instrumentation sur la substance musicale : " la musique ne réside pas tout entière dans l'utilisation plus ou moins intensive ou ingénieuse des divers instruments ni dans un amalgame inédit et plus ou moins savoureux des timbres. [...] Vingt pages de grand orchestre où s'épanouit et triomphe, à vide, la plus éblouissante virtuosité d'écriture instrumentale ne valent pas l'humble chanson d'une flûte disant seulette ce qu'elle a à dire, mais le disant bien. Pas plus que dans les discours de nos politiques, l'éclat du verbe ne réussit pas à faire passer la pauvreté du sujet... ». Ailleurs, Margat ironise sur les « engins nouveaux » qui rejoignent les rangs des orchestres (VsT 24/20).

26 «Si amusant qu'il soit de chatouiller le la naturel d'un ténor avec le si bémol d'une petite flûte ou de lui jeter à la face toutes les notes de l'arc-en-ciel harmonique, hors celle qu'il lui faut attaquer, c'est un petit jeu dont il ne faut pas abuser. Par égard tout d'abord aux chastes oreilles de l'auditeur qui, lui, n'est pour rien dans l'affaire et pourrait ne pas trouver la plaisanterie à son goût » (VsT 24/11). 
Dans une ville comme Paris, où les murs n'ont d'affiches et le badaud de regards que pour ces noms des " mille et une nuits ", riches en consonnes et longs à épeler, auxquels répondent habituellement les vedettes cosmopolites, c'est une piètre recommandation de n'avoir qu'un nom à se mettre, son nom de tous les jours, sans le moindre trait d'union, ni le plus petit y en queue.

$[\ldots]$

Lorsqu'on estime son ouvrage, on s'honore de le signer, à visage découvert. S'il n'est pas digne de soi, en sera-t-on plus fier de le signer avec un masque ? [...] D'ailleurs, le nom ne fait rien à la chose, et d'excellents maîtres de chez nous n'ont pas craint de s'appeler Durand, Dubois ou Dupont... comme tout le monde (VsT 21/6).

Margat n'est pourtant pas fermé à la musique de ses contemporains (sur laquelle ne s'empêche toutefois pas d'ironiser abondamment ${ }^{27}$ ). En se situant du côté des auditeurs, il dénonce les excès qui provoquent l'incompréhension des œuvres les moins ancrées dans le langage traditionnel. Dans un billet de 1938 ( $V_{s} T$ 25/4 $)$, il manifeste une certaine lucidité à propos des mécanismes de l'écoute, et notamment de la nécessité, pour que l'auditeur puisse suivre le discours musical et y répondre émotionnellement, d'un dosage équilibré entre nouveauté et procédés familiers (ce que Leonard B. Meyer théorisera en 1956 dans Emotion and Meaning in Music (Meyer [1956]2011)). Il est également conscient de la " signification relative qui s'attache au vocable musical » et du fait que toute la connotation d'un élément sonore (harmonique, timbral, rythmique ou mélodique) est liée à une habitude d'écoute, et que par conséquent la nouveauté ne peut susciter une réponse émotive immédiate. D'où son appel aux compositeurs à innover (ce qui est naturel) tout en tenant compte des mécanismes de l'écoute et du temps nécessaire à modifier les horizons d'attente ${ }^{28}$ :

Le Royaume de l'Harmonie a subi bien des transformations depuis $1764 \ldots$ et Rameau, son premier cartographe, ne le reconnaîtrait plus ${ }^{29}$. À l'étroit dans ses frontières, je ne dis pas naturelles, mais primitives, il a commencé, à peine érigé, de franchir les limites que son fondateur lui avait sagement assignées. Et il continue sans que rien ne laisse prévoir où s'arrêteront ses débordements. Ceux-ci font assurément moins de bruit - j'entends qu'ils font couler moins d'encre - que le déplacement des poteaux-frontière auquel on procède

27 Voir, par exemple, le billet sur l'accordage des instruments de l'orchestre considéré comme une école parfaite pour s'accoutumer à la musique contemporaine (VsT 22/26-27-28) ou celui sur l'inutilité du diapason (VsT 23/15) : " est-il bien nécessaire de feindre de se mettre d'accord avant de commencer, alors que, dès les premières mesures, il s'avère que chacun n'en fera qu'à sa tête ? "

28 Dans les mêmes années, la préoccupation d'un renouveau du langage musical sans perdre le contact avec l'auditeur était au centre de la démarche créatrice de quelques compositeurs, et notamment des membres du groupe Jeune France (dont le premier concert eut lieu le 3 juin 1936), Yves Baudrier, André Jolivet, Daniel-Lesur et Olivier Messiaen. Les écrits et les conférences de Jolivet sont particulièrement engagés dans cette réflexion (voir Lazzaro 2011).

29 La référence renvoie aux travaux théoriques de Jean-Philippe Rameau, bien qu'aucun ne soit pourtant de 1764 : le Traité de l'harmonie réduite à ses principes naturels (que Margat nomme quelques lignes plus tard) est de 1722, de 1726 le Nouveau système de musique théorique, et de 1732 la Génération harmonique, ou Traité de musique théorique et pratique; son dernier traité, le Code de musique pratique, ou Méthodes pour apprendre la musique [...] avec de nouvelles réflexions sur le principe sonore, est de 1760. 
avec tant de désinvolture pour certaines patries d'Europe... à l'étroit elles aussi. On ne mobilise pas dans le monde chaque fois qu'un dictateur hongrois, russe, voire français menace l'intégrité de la gamme diatonique ou viole les clauses les plus sacrées du Traité. Mais dans le monde pacifique des mélomanes que déconcertent le système des coups de contre-point sur la table... d'harmonie et la politique musicale du fait accompli, on ne sait plus trop où donner de l'oreille.

Le langage musical, il est vrai, n'a pas de grammaire ni de syntaxe fixes. Chacun l'emploie un peu à sa façon, ce qui ne contribue pas toujours à le rendre clair. Chaque compositeur qui se respecte l'adapte à son usage personnel. Le néologisme et le barbarisme y fleurissent à l'envi. Le malheur est qu'il faille des années pour qu'un néologisme musical devienne intelligible. Les inventeurs d'expressions nouvelles en savent quelque chose.

Avant que s'établisse une correspondance entre le terme sonore qui surprend notre oreille pour la première fois et l'ordre d'idées ou d'émotions qu'il est censé devoir suggérer, devant que ce terme encore inentendu revête un sens, s'acclimate et s'impose dans le vocabulaire, il doit se résoudre à n'être pour un temps qu'un bruit plus ou moins agréable dans sa verdeur mais parfaitement intraduisible jusqu'au jour où l'usage lui confère cette sorte de signification... relative qui s'attache au vocable musical. Mais alors d'autres formes inédites forgées dans un métal neuf viennent à leur tour obscurcir un langage auquel on commençait de se faire et dérouter une fois de plus le pauvre entendement des contemporains.

C'est ce qui explique la ferveur constante, la compréhension toujours plus vive dont témoigne le public à l'égard des œuvres consacrées... et excuse d'autre part son étonnement, sa raideur, sinon son hostilité devant les monuments insolites qu'érige, pour l'avenir, l'architecture musicale moderne. [...]

L'auditeur s'y engage... mais prudemment, à petits pas. Ne le brusquons pas... s'il a un peu le vertige ( $\underline{\text { VST 25/4 }})$.

Il y a donc une crise du public due, d'une part, aux exagérations des musiciens modernes, et, d'autre part, à un manque de culture musicale imputable aux facteurs déjà évoqués (concurrences des loisirs modernes, soutien limité à l'enseignement de la musique, etc.). Margat s'engage à combler les limites de l'éducation musicale moyenne par son activité pédagogique. Son but est d'apprendre à " l'auditeur intelligent - et c'est le nombre - [...] à écouter et à comprendre la musique comme un langage parfaitement humain et raisonnable ", de contrer le lieu commun de la musique comme " une sorte de science occulte » (VsT 22/19); dans cette perspective, il ironise sur les titres des œuvres de musique moderne qui accentuent ce mystère présumé au lieu de faire comme les vieux maîtres et laisser libre cours à l'imagination de l'auditeur :

De nos jours, le choix d'un titre se complique du fait que la musique est devenue très littéraire. Pour expliquer ce qu'elle se propose de décrire et placer l'auditeur dans l'ambiance souhaitée, il ne lui faut pas moins d'une phrase entière, sans compter les points de suspension et le sous-titre. Ce luxe de précautions ne va pas d'ailleurs sans danger. Ces titres-là sont de véritables programmes qu'on n'est pas toujours sûrs de suivre à la lettre, ou plutôt à la note. Il est plus prudent, ce me semble, de ne pas trop promettre sur un chapitre où il est quelquefois difficile de tenir et il vaut mieux laisser subsister à l'égard de ses intentions secrètes - intentions dont la pureté n'exclut pas nécessairement l'obscurité - un doute éminemment favorable à leur libre interprétation de la part du monsieur du dernier strapontin du fond dont l'imagination peut alors vagabonder sans scrupules ni remords (VsT 22/20). 
S'il insiste sur la nécessité de connaître les mécanismes de l'écriture musicale, il incite à se laisser aller au plaisir de l'écoute des nouvelles œuvres plutôt que de s' ' obstiner à vouloir d'abord et à toute force "critiquer", comparer, classer et étiqueter le plat nouveau qu'on sert » (VsT 23/7), attitude méprisable des soi-disant « connaisseurs » qui ne connaissent pas la musique mais savent en parler et qui, « de peur qu'on ne [les] y prenne à applaudir de travers, [préfèrent] ne pas applaudir du tout et se hâte[nt] de trouver d'avance tout mauvais " (VsT 25/6 ; il s'agit donc de l'attitude inverse par rapport au " complexe de Hanslick", et Margat condamne les deux en raison de leur partialité fondée sur le préjugé). On pourrait conclure que pour Margat l'apprentissage de la musique est en soi un moment d'évasion, et que la connaissance du langage musical est préliminaire au plaisir de l'écoute (car elle aide notamment à sélectionner la bonne musique susceptible de donner du plaisir), mais que la théorie apprise ne doit pas interférer au moment de la fruition en l'intellectualisant.

Dans un billet dystopique de 1936 (VsT 22/25), Margat imagine le jour où un peintre sera toujours à côté du musicien pour illustrer la musique qu'il joue au bénéfice d'un auditoire " profane " incapable de saisir le langage musical dans un monde où la vue s'impose. Margat réagissait probablement aux Cinéphonies, courts métrages musicaux produits entre 1935 et 1938 par la Compagnie des grands artistes internationaux (avec la participation des réalisateurs Marcel L'Herbier, Dimitri Kirsanoff, Max Ophuls et la supervision de l'influent musicographe Émile Vuillermoz) ${ }^{30}$. Le lecteur d'aujourd'hui ne peut pas s'empêcher de reconnaître dans les fantaisies de Margat des réalités aujourd'hui devenues habituelles, des vidéoconférences aux diaporamas qui accompagnent les présentations orales, des vidéo-clips aux graphismes générés par les logiciels reproducteurs de musique :

Aujourd'hui, on ne vous croit plus sur parole. On demande à " voir ».

Bientôt, au téléphone, on ne se contentera plus de percevoir, à tant la minute, l'inflexion d'une voix chère. On exigera, pour le même prix, de contempler à l'autre bout du fil la bobine de son interlocuteur. Vision, ou plutôt... télé-vision fugitive qui, lorsqu'il s'agira d'une interlocutrice jeune et jolie, donnera un agrément certain à la conversation.

Sur l'estrade, le conférencier à la page, dans son feuilleton, le chroniqueur à la ligne ne pourront désormais se faire comprendre sans le secours, le premier, d'un tableau noir, le second de hors-texte explicatifs.

Des mots... des mots... des mots !... dit Hamlet ${ }^{31}$.

Des notes... des notes... des notes !... soupire, au concert, le chœur profane des auditeurs devant l'énigme de plus en plus indéchiffrable d'un langage sacré, mais obscur, dont la clé leur échappe et qu'ils ne savent pas lire entre les lignes. Alors, comme aux petits enfants ou aux grandes personnes un peu dures d'oreille... ou d'entendement, on leur fera une figure. Une pianiste ne se risquera plus sans son peintre, qui sera chargé « d'extérioriser » sur une toile, en rose ou en bleu, en long ou en large, la courbe sinueuse d'un thème romantique, l'éblouissement d'un arpège, le bouillonnement des harmonies et, d'une façon générale

30 Voir Heu 2015, vol. 2, p. 10, 38, 226, 422. Le Guide du concert avait réagi très favorablement aux Cinéphonies à travers la plume de son directeur, Gabriel Bender (1936).

31 "Words, words, words ", répond Hamlet à Polonius qui lui demande ce qu'il est en train de lire (William Shakespeare, Hamlet [1599-1601], II, 2). 
et définitive, de traduire à grands coups de pinceau ou de fusain le sens caché des textes qu'elle débitera sur le clavier (VsT 22/25).

\section{NOSTALGIE D'UN MONDE SONORE QUI N'EST PLUS}

La capacité de sélectionner la bonne musique afin de pouvoir en tirer plaisir est encore plus urgente dans un contexte de surmusicalisation du quotidien (par la radio et les disques) et d'intensification des bruits ambiants à l'ère des machines. Il en découle, chez Margat, une véritable convoitise du silence qui s'exprime sous la forme d'une nostalgie du silence perdu (le "vrai silence, [le] silence d'avant-guerre, [1e] silence cent pour cent " ( $\left.\left.V_{s} T 23 / 8\right)\right)$. Dans la forêt de Fontainebleau, écrit-il dans un de ses premiers billets, on n'entend presque pas d'oiseaux, sauf « l'appel criard du geai solitaire ou, sur le soir, le croaillement [sic] d'une corneille épeurée » $(\underline{V s T} 21 / 5)$. Mais cela ne signifie pas que la forêt soit silencieuse :

La forêt sans oiseaux... refuge suprême du divin silence ? Las ! N'y comptez pas!

Du ciel bleu qu'emplit le vrombissement incessant des avions, de la route proche où l'avertisseur hurle à la mort, du champ de tir voisin où la mitrailleuse d'instruction ponctue, à blanc, je ne sais quelle diabolique dictée musicale - un triolet, deux croches, une noire les bruits que vous pensiez fuir vous poursuivent, vous rejoignent et vous accompagnent sans que vous réussissiez jamais à les dépister tout à fait.

Silence des bois inhabités...

Silence de l'âme moderne. La forêt sans oiseaux... telle je m'imagine la cité future où, les dernières ariettes oubliées, le dernier rossignol envolé, nos petits neveux n'auront pour distraire leur ennui et charmer leurs loisirs que le fracas des machines et le grincement des mécaniques $^{32} \ldots$ (VsT 21/5).

La disparition $\mathrm{du}$ silence est un thème qui revient régulièrement dans les "Variations... sans thème ». En plus des bruits artificiels qui couvrent les sons de la nature, Margat regrette à plusieurs reprises la musicalisation du quotidien par la radio (la « radiocacophonie » (VsT 24/10)), surtout lorsqu'on la subit de façon involontaire :

Ma concierge a la TSF. Libéralement, elle en régale la maisonnée. Par la grâce du toutpuissant et très-haut-parleur qu'elle possède, l'Harmonie, copieusement distribuée à tous les étages, avec l'eau, le gaz et l'électricité, règne en souveraine de l'entresol au cintième [sic]. Mais gratis. Seulement, je ne peux pas, à mon gré, fermer le compteur. Alors, l'été, je ferme la fenêtre.

Remarquez que je ne conteste pas du tout à une concierge amie des arts le droit absolu de disposer des ondes chantantes et parlantes. [...] Je souhaiterais seulement qu'en bas, comme d'ailleurs chez le locataire du dessus et chez la dame sur la cour, on apportât plus de pudeur à la consommation, en famille, du péché de Radiophonie et qu'on gardât plus jalousement ses extases pour soi.

32 On pourrait se demander si la « forêt sans oiseaux » de Margat est un clin d'œil à une célèbre formule tirée de La vie littéraire (1888-1892) d'Anatole France, selon laquelle « sans l'ironie, le monde serait comme une forêt sans oiseaux ». En ce sens, l'ironie des billets de Margat serait-elle à interpréter comme un remède contre la mécanisation du monde qu'il dénonce. 
$[\ldots]$

Maintenant, vous me direz qu'il y a des gens que le haut-parleur du voisin n'empêche pas de dormir, de penser, de lire, de travailler, ni même de chanter... autre chose - ce qui est un bel exemple d'indépendance ou de surdité musicale ( $\underline{\text { VsT 22/4 }}$ ).

Dans le texte qui ouvre la deuxième année des «Variations... sans thème » (vol. 22 du Guide du concert, 1935-1936), l'opposition son naturel/son artificiel subit un glissement par rapport au billet sur « la forêt sans oiseaux ». Ce n'est plus l'origine du son qui en détermine la nature « naturelle» ou artificielle, mais l'habitude de l'écoute. Ainsi, la " chanson du rail » d'un train en cours est classée parmi les sons naturels que la nouvelle Radio-Fer, la radio des chemins de fer, vient à déranger ${ }^{33}$. La radio tue les temps morts, occupe chaque instant et contribue à l'accélération de la vie, provoquant chez Margat une nostalgie du temps lent, du silence (qui peut même être bruyant, s'il s'agit du bruit naturel du moyen de transport) et de ce que cette absence de musique ambiante pouvait susciter autrefois, des rêveries aux conversations aux idées musicales :

Avec la Radio-Fer, on peut dire que la « musicalisation » - à vous, mon cher Bender ${ }^{34}$ - va son train.

La Radio-Fer - pour la foule des déshérités qui en sont réduits à faire la route sur les quatre pneus d'une "familiale », que la sévérité des temps force à conduire eux-mêmes - la Radio-Fer désigne, simplement, la TSF en chemin de fer.

Plus les voyages s'abrègent, plus on s'ingénie à en tromper l'ennui. Nous arrières-grandsparents [sic] qui, de reste, n'étaient guère nomades et n'étaient point si pressés que nous d'arriver, n'avaient, pour charmer les interminables heures de diligence, que l'agrément des conversations, le ronron berceur du voisin assoupi ou, peut-être, de lieue en lieue, le refrain d'une tabatière à musique, tirée hors du gousset par quelque gentilhomme mélomane ou facétieux. On sait mieux, maintenant, le prix du temps. Il n'est pas de trop brève occasion de s'instruire et il faut louer sans réserve l'éclectisme du dispensateur d'émotions artistiques qui, du fourgon de queue, vient éveiller parmi le fracas des aiguillages, le subconscient musical qui sommeille au fond de chacun de nous.

Pour ma part, toutefois - mais c'est un regret tout personnel -, j'aimais mieux le temps encore tout proche où, blotti dans mon coin, côté fenêtre et bercé durement par la chanson $\mathrm{du}$ rail, je composais sans fin des thèmes et variations qui tous - c'est assez vexant à avouer - avaient un rythme de polka ${ }^{35} \ldots$ (VsT 22/1-2).

33 La Société Radio-Fer a inauguré l'installation de la TSF sur les trains le 8 février 1930, sur la ligne Paris-Le Havre ([Anonyme] 1930a). Son président, Jacques Bompard, a été promu officier de la Légion d'honneur en juillet ([Anonyme] 1930b).

34 Gabriel Bender était le directeur du Guide du concert (voir sa fiche biographique ici : http://pressemusicale.oicrm.org/corpus/musicographes). En 1930, en ouvrant l'enquête « Audition directe et audition indirecte », il avait décidé de limiter l'analyse de cette dernière au seul phonographe, en écartant la radio ([Bender] 1930). Il est à présumer que Bender n'était pas favorable à la " musicalisation » : avant et après l'avènement de l'enregistrement et de la radiodiffusion, il a régulièrement défendu dans ses articles l'écoute non distraite et non simplement " physiologique " (voir par exemple, Bender 1913 ; Bender et Rousseau 1928).

35 La boutade finale assume, rétrospectivement, une nuance ironique. En effet, entre 1940 et 1944, Margat composera, sous le pseudonyme d'Ivan (ou Yvan) Marfel et en collaboration avec Claude Aral 
La position «technocritique» de Margat ne doit pas être comprise comme étant la plus courante à l'époque ${ }^{36}$. Qu'on la compare, par exemple, à l'enthousiasme de cet article anonyme du Petit Journal, où la valeur accordée à l'ennui et au temps lent du voyage par le musicographe est renversée en contrainte indésirable que la technologie peut finalement abolir :

Ah ! la monotonie des longs voyages en chemin de fer! Quels que soient la beauté ou l'imprévu des paysages que vous voyez défiler, quel que soit l'intérêt des livres ou des revues que vous avez emportés, quel que soit l'attrait de la conversation de vos voisins, il arrive toujours un moment où l'ennui vous gagne irrésistiblement. Eh bien, voyageurs de demain, apprenez cette bonne nouvelle : bientôt, vous ne vous ennuierez plus dans le train. Deux écouteurs aux oreilles, vous pourrez entendre des concerts, des conférences, des informations. [...] Bref, un voyage ne sera plus, comme aujourd'hui, un hiatus dans votre vie ([Anonyme] 1930a).

Trop de stimulation sonore, dénonce Margat dans un billet exploitant de façon très puissante et virtuose l'énumération, conduit, si ce n'est pas à la surdité, à tout le moins au fait qu'on n'écoute plus :

Faites la somme des bruits divers qui dans la grand'ville nous heurtent le tympan du lever au coucher - sans parler du tapage nocturne -, additionnez tout ce qui résonne, chante, vocifère, braille, gronde, siffle, ronfle, bourdonne, éclate et détone autour de nous du matin au soir et vous obtiendrez une minute de tintamarre auprès duquel le fracas du Niagara n'est qu'indistinct murmure et doux chuchotement.

Le miracle est que nous ne soyons pas encore sourds. À vrai dire, si l'on entend encore, il y a belle lurette qu'on n'écoute plus. Dans nos maisons pleines de TsF qui s'égosillent, de machines à écrire qui crépitent, de téléphones qui carillonnent et de portent qui claquent ; dans la rue où l'avertisseur hurle à la mort parmi les ruades et les pétarades des chevauxvapeur ; dans le grand magasin où la Prière de la $\mathrm{TosCa}^{37}$ vous poursuit jusqu'au rayon de savons à barbe ; à la Chambre où chaque orateur s'exténue à se faire entendre sans parvenir à se faire écouter ; au concert enfin où les instruments de l'orchestre s'évertuent simultanément à jouer chacun leur petit air dans un ton différent ; qu'il s'agisse de mélodies susurrées par Mlle Y... de l'Odéon, entre la poire et le fromage ; de faux numéro de l'inter [le téléphone]; de passages cloutés où l'on se risque quand même; du débat sur la dévaluation de... la France ou de la 1 ère audition d'un jeune compositeur letton, on finit par ne plus savoir ni ce qu'on entend, ni ce qu'on dit... ni, hélas ! ce qu'on fait.

$\left.{ }^{* 38}\right]$ [satu?]ration sonore que nous ne tarderons guère à ne plus pouvoir distinguer, à

(pseudonyme de Lucie Soufflet?), une longue liste de chansons sur rythmes de danse (java, fox-trot, tango, etc.). La liste est accessible à cette page de recherche dans le catalogue de la Bibliothèque nationale de France (BnF) : https://goo.gl/UjHBXS.

36 Pour une étude des discours « technocritiques », voir Jarrige [2014]2016.

37 Margat avait déjà évoqué la nuisance de la Prière de la Tosca de Giacomo Puccini («Vissi d'arte, vissi d'amore », II, 5) dans un précédent billet : « Il y a des moments dans la vie où la Prière de la Tosca [...] vous semble particulièrement inopportune. Et il y a vraiment des Tosca qui exagèrent » (VsT 22/4 $)$.

38 En raison d'un problème de mise en page, la première ligne du paragraphe a été substituée par une ligne appartenant au précédent (" [dif-]fèrent ; qu'il s'agisse de mélodies susurrées »). À remarquer : dans cette reprise erronée de la ligne, le mot «susurrées » est écrit correctement avec un seul s, tandis qu'avant 
l'audition, un do d'un $f a$, une chanson d'un monologue, la prose des vers, le final d'une symphonie contemporaine de la dégringolade d'un vaisselier, le français de l'iroquois et une harangue de M. Mussolini d'un discours du chancelier Hitler...

Alors, l'abus de la fonction déformant l'organe, nos arrière-petits-neveux, dans un temps plus ou moins proche, verront s'allonger, de chaque côté de leur crâne définitivement vide, une magnifique paire d'oreilles d'âne... (VsT 23/11).

Dans cette époque d'audition sans écoute, " le Français moyen peut désormais s'enorgueillir d'un nouveau titre : celui d'Auditeur » (VsT 23/17). Il est, de surcroît, un "bon auditeur » (tel qu'il est « bon citoyen, bon époux, bon père de famille »), il écoute « docilement [...] tout ce qu'on veut » (et Margat nous régale d'une nouvelle énumération, portrait narquois de la programmation radiophonique, $\mathrm{du}$ " bulletin météorologique lui annonçant le temps qu'il fit hier " au cours d'espéranto, de la leçon de culture physique au " quart d'heure du jardinier ", " le tout largement arrosé de romances des meilleurs crus", ibid.). Le billettiste invoque alors l'institution, sinon d'un " ordre des auditeurs ou d'une médaille des bons auditeurs », au moins du " titre d'Auditeur de $1^{\text {re }}$ classe » donnant droit au besoin le plus pressant de Margat : un jour de congé par semaine (ibid.) ${ }^{39}$.

Le discours de Margat se situe dans une recherche des causes de la crise de la musique : il regrette le temps perdu ou l'on pouvait se permettre de prendre (et perdre) son temps, où l'absence du diktat de l'efficacité pouvait favoriser l'inspiration musicale, seul moteur, à ses yeux, de la bonne musique. Margat dénonce le nombre de compositeurs qui manquent d'inspiration (mélodique, notamment), ce qui pour lui équivaut à ne pas être "doué pour la musique ». Dans un billet burlesque, le musicographe raconte les dons de mélodiste du peintre en bâtiment venu travailler dans son nouvel appartement en y apportant " non seulement la peinture, mais encore la musique, [...] et la musique en ce qu'elle a de plus jaillissant, de plus intuitif. J'ai nommé la Mélodie» :

Si la verve mélodique est la marque du génie musical, mon petit peintre, une fois juché sur son échelle double, se révélait grand musicien. Il peignait. Après une romance, une romance encore. Ce qu'il ne chantait pas il le sifflait (VsT 22/8).

De cet exemple surgit l'appel moqueur de Margat :

Compositeur[s], mes frères, quand l'inspiration vous fuit et que vous séchez, le crayon en arrêt, devant votre papier rayé où les bribes d'un thème récalcitrant refusent de se rejoindre, n'insistez pas. Rangez votre écritoire, louez une échelle, empoignez un pinceau et si, là-haut, la Mélodie ne ruisselle pas avec l'huile de lin qui ne manquera pas d'inonder

il en avait deux. On se demande donc s'il ne s'agirait pas de deux paragraphes alternatifs (la référence à Mussolini et Hitler pourrait laisser croire que ce passage ait été retenu non publiable), corrigés par Margat sur les épreuves et ensuite mal composés par le maquettiste.

39 Dans le premier billet de l'automne 1938, Margat fait l'éloge du « silence hygiénique et réparateur auquel tout citoyen-mélomane devrait avoir droit [...] quinze jours chaque année » pendant les vacances estivales (VsT 25/1-2). 
votre manche, c'est qu'alors vous n'êtes pas doué pour la musique. Dans ce cas, vous feriez mieux de faire de la peinture... (ibid.).

L'inspiration étant pour Margat le moteur essentiel de la création, il dénonce l'intérêt philologique pour l'étude du processus créateur. Dans le billet transcrit ci-après, l'humanisation du compositeur-génie, en l'occurrence de Beethoven, est dogmatiquement réprimée par Margat, qui ne peut admettre que le génie rate et corrige :

Un manuscrit attribué à Beethoven vient d'être exhumé. Il s'agirait de l'immortelle Messe en ré. Ce grimoire rarissime étonne, nous dit-on, par sa profusion des ratures, corrections et surcharges qui en couvrent les feuillets jaunis, témoignant du soin pointilleux qu'apportait le génial artisan au parachèvement de son œuvre.

Eh bien! Si la chose est authentique, voilà un secret de fabrication qu'on ne devrait pas divulguer. Il n'ajoute rien au prestige du grand musicien, au contraire. L'honnêteté, le scrupule, l'application sont petites vertus, vertus de deuxième zone, bonnes pour les besogneux et qu'on ne saurait prêter sans offense au génie. L'idée d'un Beethoven penché sur sa partition comme un clerc d'étude sur son dossier et fignolant, le grattoir en main, le contour d'un premier violon ; d'un Beethoven hésitant, tourmenté, perplexe, y regardant à deux fois avant d'écrire une note, en proie au scrupule de conscience, biffant à grands coups de plume d'oie la demi-page de la veille, cent fois sur le métier remettant son ouvrage, le polissant sans cesse et le repolissant, cette idée-là va à l'encontre de toutes les idées reçues. Parlez-moi d'un homme qui, la redingote au vent, le chapeau sur la nuque et les mains derrière le dos, s'en va, par une romantique après-midi d'automne, à travers la campagne, ramasser une pleine brassée de thèmes et rentre, le soir, avec un mouvement de symphonie qu'il n'a plus qu'à coucher par écrit. À peine prend-il le temps de s'asseoir. Le voici devant son écritoire. Il compose. Après une feuille noircie, une autre feuille noircie. Sa plume court, fiévreuse, couvrant la page de pattes de mouches désordonnées mais définitives, escaladant les portées, négligeant dans sa folle randonnée de barrer les croches et d'accrocher les dièzes [sic] à la clé. L'Esprit souffle en tempête, ses rafales se succèdent sans répit, les idées jaillissent, fusent, se superposent, s'enchevêtrent, s'affrontent ou s'ajustent harmonieusement dans l'équilibre parfait d'un plan magistral, réalisé d'un seul jet, d'une seule haleine, d'une seule encre, jusqu'à la strette finale et le dernier soubresaut de la chandelle agonisante...

Voilà comment naissent les chefs-d'œuvre, les vrais. Quant à ce soi-disant manuscrit laborieux de la Messe en ré, ce ne peut être que l'œuvre d'un méchant gratte-papier de l'époque ${ }^{40}(\underline{V s T} 23 / 18)$.

40 Le manuscrit qui fait l'objet du billet fait probablement partie du recueil de sources beethoveniennes conservées à la bibliothèque du Conservatoire de Paris décrites par Julien Tiersot dans La Revue de musicologie en 1927 (Tiersot 1927) et ayant été exposées à l'occasion du centenaire de la mort du compositeur. Margat aurait pu le voir à cette occasion (donc une dizaine d'années avant l'écriture du billet, ce qui semblerait cependant en contradiction avec le "vient d'être exhumé » qui ouvre le texte). Plus particulièrement, il pourrait s'agir des esquisses de la Messe en do qui avaient été faussement identifiés, dans l'exposition, à la Messe en ré, tel que précisé par Tiersot (ibid., p. 72, n. 1). Sur les sources manuscrites de la Missa solemnis, voir Kinsky-Halm 2014 (ad vocem) ; Virneisel 1966 ; Winter 1984. 
Cet idéalisme romantique presque caricatural contraste d'ailleurs avec d'autres textes où Margat insiste au contraire sur le rapprochement entre musiciens et public : lorsque, tout en louant les biopics comme d'excellents moyens pour « la propagande musicale populaire ", il les accuse d'exagérer le génie des compositeurs, qui sont au fond des bons bourgeois " comme vous et moi ", attentifs à leur revenu (VsT 21/23); ou lorsqu'il humanise les interprètes, qui souffrent de " trakéite " (scilicet, ils ont le trac) et qu'il faut donc encourager (VsT 21/24), et qui ont des contraintes très prosaïques comme celle de ne pas rater leur train après le spectacle (ne leur demandez donc pas de bis ! [VsT 21/20]) ${ }^{41}$.

Plus de silence, plus d'inspiration, tout change, et Margat se dresse à témoin sarcastique du changement. Un billet de 1938 réunit dans une fresque hilarante tous les éléments de la vie moderne qui contribuent à modifier la vie musicale domestique : les nouveaux loisirs - surtout sportifs - des jeunes filles les éloignent du piano ${ }^{42}$, qui devient un meuble muet (ou maltraité) remplacé par la radio et le phonographe sollicités pour jouer les nouvelles danses remplaçant du coup les morceaux des classiques :

Si l'on danse encore en famille, ce n'est certes plus la faute du piano. Depuis l'avènement du pick-up et de la radio, il n'a aucune part aux sauteries d'à présent et le mutisme renfrogné où il s'enferme, dans son coin, dit assez sa réprobation d'un sport qui n'est plus de son âge. Le rôle de jeune fille de la maison s'en trouve bien simplifié si l'on considère la contribution artistique qu'elle était censée apporter naguère au succès des soirées dansantes dont nos mères avaient le secret. Il se réduit désormais à remonter la mécanique du phono et à changer la face du disque. Aujourd'hui la jeune fille fume, confectionne le cocktail maison, y goûte à l'occasion, raconte la dernière histoire marseillaise. Elle sert encore le thé aux personnes âgées mais ne joue plus du piano. Le ski, le crawl, le droit international, voire la pharmacie ont remplacé dans les préoccupations de nos modernes jouvencelles les arts d'agrément qui faisaient autrefois le plus gracieux ornement d'une demoiselle accomplie au sortir du couvent : la broderie anglaise, la pyrogravure, l'aquarelle, la musique et les confitures. [...]

Ce sont maintenant les fox et les rumbas qu'on détient en conserve dans les casiers de la discothèque. C'est un dessert tout trouvé et qui, tout compte fait, revient moins cher que le Schubert et le Chopin préparés chez soi. Quant au vieux piano d'héritage qui dans un angle obscur du salon drape sa dignité et son vernis noir dans les plis flatteurs d'une soierie du Japon, il doit se résigner, avec les gens de sa génération, à faire tapisserie. On le garde par déférence, comme un vieux parent perclus qu'on n'a pas l'impudence de reléguer à la maison de retraite. Mais il y a belle lurette qu'on ne se soucie plus de lui, sinon pour l'épousseter de temps à autre d'un plumeau distrait et pour renouveler les fleurs de ses vases $^{43}$.

41 Sur l'aversion de Margat pour la pratique du bis, voir également VsT 23/35.

42 Dans un autre billet (VsT 25/7), Margat se demande quels instruments sont les moins appropriés pour une jeune fille (le trombone à coulisse, " à moins d'y être contrainte par la dureté des temps » et le saxophone, " cette sorte d'énorme pipe avec quoi de charmantes jeunes filles se risquent à s'afficher en public n'est vraiment pas de leur âge ni de leur sexe »), pour en conclure que de toute façon « les demoiselles d'aujourd'hui préfèrent le volant».

43 Sur l'état épouvantable des pianos dans les maisons bourgeoises, voir aussi VsT 22/10 et VsT 21/21: 
Pourtant, dans l'abandon général, voici qu'ici une voix innocente s'élève en sa faveur. Celle de la petite dernière qui va sur ses quatre ans et tourne autour du vieux meuble dont la forme bizarre et l'inutilité flagrante l'intriguent. Un jour, elle a soulevé le couvercle et posé curieusement trois petits doigts potelés sur une touche jaunie. Cela a déclenché soudain, à l'intérieur du vaisselier à bougeoirs, la chute d'une timbale fêlée ou d'une cuiller à café, on ne sait pas au juste. La coupable, extasiée, a promené alentour un regard triomphant. "Elle sera musicienne ", a déclaré le père. "Quand elle aura six ans, nous lui ferons commencer le piano ", a opiné la mère... qui ne se souvient plus. Et bientôt les premières gammes ont commencé de trottiner comme des souris blanches et noires sur le clavier réhabilité.

Les années ont passé. Bébé vient d'avoir dix-huit printemps. On fume, on connaît la recette du dernier cocktail et les derniers potins d'Hollywood. "Que désires-tu pour ton anniversaire, ma chérie ?", lui demanda sa maman. Et l'enfant blonde de lui répondre, sans hésiter : «Un phono!» (VsT 24/19).

L'insistance de Margat sur la décadence que représenterait la substitution de la pratique instrumentale par l'écoute radiophonique ou phonographique n'est pas uniquement une forme de technocritique ni une dénonciation de l'écoute distraite. Le billettiste-professeur d'harmonie est réellement convaincu, nous l'avons vu, de l'importance de l'étude de la musique et en souligne plus particulièrement le rôle indispensable dans l'éducation des jeunes. L'éducation musicale est pourtant méconnue par l'État (la musique, à la différence de l'art, n'est pas une matière dispensée gratuitement au lycée ${ }^{44}$ ) et est de plus en plus délaissée par les familles bourgeoises en raison de l'accès facile aux enregistrements :

La TSF qui multiplie dans tous les milieux l'occasion d'entendre de la musique est en train d'ôter à toute une génération le désir ou... le courage d'en faire. [...]

Alors, il resterait à convaincre les familles de nos petits Français que la Musique n'est pas seulement un art d'agrément, mais encore et surtout un moyen d'éducation ; qu'elle a, dans la formation intellectuelle et morale de la jeunesse, un rôle de premier ordre à remplir ; que le phono ni la TSF ne sauraient remplacer l'effort personnel et fécond que réclament la pratique, même malhabile, d'un instrument et le déchiffrage, même laborieux, d'une page musicale ; que la musique est une école de discipline, de logique et d'équilibre et qu'il n'est pas besoin d'y passer maître pour recueillir le bienfait de son enseignement...

Et voilà pourquoi, si votre fille est muette devant son poste TSF, il faut lui apprendre à chanter... elle aussi. Voilà pourquoi, à côté du pick-up, dans la salle à manger familiale, le piano a toujours sa place, ou l'étui à violon. Avec un violon dedans, bien entendu ( $V s T 25 / 9)$.

Quel antidote au changement Margat propose-t-il ? En pratique, il s'investit personnellement dans ses cours d'harmonie pour combler les vides qu'il dénonce. En ce sens,

«On les conserve pieusement, en souvenir, par esprit... de famille et... d'économie. Ça ne tient plus l'accord depuis 30 ans ; la pédale, elle, tient toute seule, en permanence. Les cordes, détendues et rongées par la rouille, exhalent un son de tringle à rideau quand un marteau mité parvient, par hasard, à en frapper une. Un bon tiers des "notes" ne parlent pas et c'est toujours ça de gagné pour l'oreille. Mais "le meuble garde tout de même sa valeur", "c'est de l'acajou véritable, Monsieur" ".

44 Voir VsT 24/3. 
on peut considérer ses activités pédagogique et musicographique comme intimement reliées : non seulement, comme nous le soulignions au début du présent article, la publicité des cours de Margat dans les pages du Guide du concert contribue à donner crédit à sa parole écrite et, réciproquement, sa visibilité de billettiste hebdomadaire devrait lui attirer davantage d'élèves (payants ou du cours préparatoire gratuit réservé aux lecteurs du Guide), mais, qui plus est, ses cours sont l'antidote à la crise dont il trace la caricature. Dans une interview signée P. V. publiée dans Le Guide du concert à l'automne 1936 (en concomitance avec la reprise de ses cours : il s'agit donc bel et bien d'une réclame, d'autant plus que Margat est en couverture du numéro (figure 5)) ${ }^{45}$, Margat s'exclame que « le meilleur moyen d'amener des adeptes à l'harmonie, c'est de la faire connaître » (P. V. 1936); dans sa «Lettre à l'auditeur... inconnu » publiée " à l'occasion de l'ouverture de la $6^{\mathrm{e}}$ session de son cours préparatoire d'harmonie qui suscite parmi les lecteurs de cette revue un intérêt croissant d'année en année ", il vante l'accessibilité de ses cours et leur formule non académique. Son Cours pratique d'harmonisation et d'accompagnement au piano, précédé d'un Abrégé de l'harmonie classique publié beaucoup plus tard chez Durand (Margat 1953) répond au même souci de vulgarisation de l'harmonie dans une clé différente par rapport aux traités ${ }^{46}$ :

Ce cours s'adresse à la fois aux pianistes qui se proposent d'aborder l'accompagnement mais n'ont pas encore commencé l'étude de l'harmonie et à ceux qui, possédant déjà des notions théoriques de cette science, souhaitent d'en faire l'application pratique à l'harmonisation au piano (Margat 1953, « Avertissement », c'est l'auteur qui souligne).

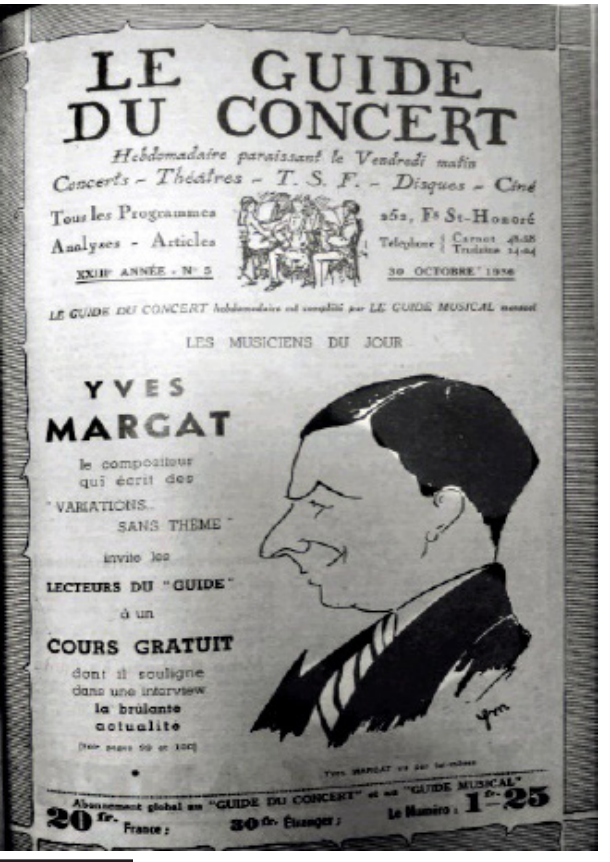

Figure 5. Couverture du Guide du concert, vol. 23, $n^{\circ} 5$ (30 octobre 1936), avec une autocaricature d'Yves Margat et l'annonce de l'interview où il souligne "la brûlante actualité » de son cours d'harmonie.

45 Margat et ses cours occupent la couverture du Guide du concert à tous les automnes à partir de 1935 (la deuxième année des «Variations... sans thème »). Dans le premier numéro de l'année précédente, une photo de ses cours (lui au piano, une foule d'élèves souriants tout autour) est par ailleurs publiée dans les pages des annonces (vol. 21, n⿳ 1, p. 35). Et vers la fin de l'année (avril 1935), la revue consacre au billettiste-professeur sa première couverture, à l'occasion de la publication d'un compte rendu élogieux d'une séance de ses cours (X. 1935).

46 En 1941, Margat avait aussi écrit un Traité de l'harmonie classique (Margat 1941 ; Réalisations des exercices parues dix ans plus tard). Les exercices préparés pour ses cours d'harmonie sont eux aussi publiés (deux séries d'Exercices préparatoires à l'étude de l'harmonie parues chez Durand en 1936 et 1939 respectivement). 
Sur le plan du discours, en revanche, Margat ne propose pas de véritables solutions (par exemple, en cherchant, comme d'autres critiques le font, à envisager un rôle positif pour la musique enregistrée) et préfère se réfugier dans une évocation sublimée et nostalgique d'un monde qui n'est plus. Un monde dont il idéalise même la musique légère, que les arrangements modernes et leur diffusion radiophonique insulteraient ${ }^{47}$. Un monde habité par des figures musicales disparues, comme le joueur de serpent (VsT 21/9) ou le souffleur d'orgue que la technologie a remplacé (" avant que la race des souffleurs soit à jamais éteinte et que le ronflement du moteur électrique ait définitivement remplacé [...] l'ahan du brave moteur à bras » $(V s T 23 / 22))$. Un monde de façons d'être en société disparues elles aussi : Margat intervient à plusieurs reprises sur les changements dans le domaine de l'habillement (VsT 23/16 ; 24/31-32) et ridiculise la mode d'imprimer des symboles musicaux et des fragments de partition sur les foulards des dames - dernier rempart de la présence de la musique en société ( On ne dira plus que la mode féminine manque de mesures, ni que ses manifestations demeurent sans portées!»(VsT 25/11-12-13)). Surtout, Margat investit de nostalgie le monde de l'enfance. De son enfance, d'abord ( $V s T 23 / 20)$, mais plus généralement de l'enfance comme catégorie non contaminée par la crise, et qui amène un peu de fraîcheur nonobstant le massacre de la musique que les jeunes mains provoquent (VsT 23/21;23/27-28).

Il y a de l'espoir, selon lui, à constater que parmi les cadeaux de Noël présentés dans un catalogue de 1937, il n'y a pas seulement " un magnifique camion-pétrolier, éclairage électrique, et un séduisant tank à feu surmontant obstacle et lançant projectiles », mais aussi des jouets musicaux classiques (« l'ours-humoristique-cymbalier et la pêche-miraculeuse-à-musique » ou « le petit piano laqué d'une octave [...] qui naguère, avec le cheval mécanique à trois roues, excita vainement ma convoitise plusieurs Noëls durant ») ou modernes (" un jazz sur pied métal [...] qui remplace avantageusement feu l'homme-orchestre » et surtout " un superbe phonographe-valise, façon cuir, roi incontesté de ces instruments-jouets »(VsT 23/12-13-14)). En outre, Margat voit favorablement le fait que les enfants sont (peut-être les seuls) encore attirés par le piano du salon autrement fermé (comme dans le billet transcrit plus haut, VsT 24/19). Malheureusement, les nouvelles méthodes de pédagogie musicale semblent menacer cet âge encore pur : le «sport » envahit même l'apprentissage de la gamme, qui devient une " petite gymnastique musicale et, par ailleurs, hygiénique » (on reconnaît, dans la description découragée de Margat, la méthode Kodály ${ }^{48}$ ):

47 «Valses alanguies, polkas piquées, gais flonflons des quadrilles 1900, et vous, romances enrubannées qui datez du temps des cerises, ariettes oubliées dont on fit un paquet, voilà quelque trente ans, et qui moisissez sur une planche du grenier [...]. C'est vous qui ce soir faites les frais ou avez les honneurs de ce radio-concert consacré [...] aux succès d'autrefois. Car ce n'est pas le vieux piano à colonnades et à bougeoirs de votre jeunesse qui ressuscite ainsi vos grâces surannées. [...] C'est autre chose, quelque machine inconnue [...]. Et comme on vous a parés pour la circonstance ! Que vous voilà pimpants et coquets ! Écoutez-moi cette orchestration, ce rythme, ce saxo et jusqu'à cette batterie discrète [...] » (VsT 25/20).

48 Sur l'adaptation de la méthode Kodály en France, voir Spire 1997. 
Apprendre à grimper en haut d'une gamme est à présent toute une histoire. [...] Cela consiste en une série de manières de petits saluts fascistes. Il y en a huit : un par note, qui s'échelonnent de la ceinture au chignon en passant par l'épaulette et la jugulaire (VsT25/3).

\section{CONCLUSION}

Les "Variations... sans thème " sont une source d'informations à la fois riche de contenu et légère dans le ton pour l'étude du discours sur la musique à Paris dans la difficile période qui a mené à la victoire du Front populaire et, ensuite, à la Seconde Guerre mondiale. En raison de leur nombre, elles constituent un barème précieux sur des sujets sensibles de l'époque - les effets de la crise et de la modernité envahissante sur la musique qui génèrent, par contraste, la construction discursive de la " belle époque » d'avant-guerre (époque de la jeunesse de Margat) ${ }^{49}$.

Comme dans tout cas de microhistoire, il est difficile de déterminer jusqu'à quel point il est possible d'étendre les idées, les perceptions et les nostalgies de Margat à ses contemporains - ou, pour utiliser une formule à calembours, jusqu'à quel point ses représentations sont représentatives. Il faut toutefois souligner que la médiatisation de ses positions en fait, sinon un reflet, potentiellement un modèle pour son public de lecteurs. D'où l'importance de contextualiser sa prise de parole en la reliant à la visibilité que Le Guide du concert offre à ses cours d'harmonie, ce qui permet d'inférer l'autorité que les mots du billettiste-professeur pouvaient avoir sur ses lecteurs/élèves. Compositeur sans succès ${ }^{50}$, sans position dans l'enseignement officiel, Margat se fait le porte-parole des autres musiciens de profession qui n'ont pas sauté dans le train de la modernité et qui se renferment donc, de façon à la fois méfiante et auto-justificative, dans un conservatisme à propos des positions esthétiques et du goût qui leur donne au moins l'appui sympathique du large public d'amateurs dérouté par les nouvelles tendances. La lecture intégrée des "Variations... sans thème » avec la visibilité publicitaire donnée aux cours de Margat par Le Guide du concert laisse croire que cette action de soutien réciproque entre les propos exprimés par Margat et son dévouement pour la vulgarisation des secrets de l'harmonie aurait pu, à moyen terme, servir la cause du Margat aspirant compositeur : un public formé pour apprécier la musique qu'il considère digne d'écoute pourrait, en effet, être porté à aimer la sienne. Selon cette grille de lecture, le statut même des billets de Margat serait à redéfinir : ils ne seraient pas seulement des prises de parole d'un faiseur d'opinions humoristique, mais une véritable poétique à feuilletons d'un compositeur. Un musicien qui, en dépit de pouvoir présenter ses œuvres, assure sa présence sur la scène musicale en composant des « variations » verbales.

49 L'affirmation du concept de Belle Époque remonte effectivement aux années 1930, comme l'a démontré Dominique Kalifa (2017).

50 Les compositions publiées de Margat se comptent sur les deux mains : trois pièces pour piano publiées par les éditions Émile Gallet et fils en 1932 (Dans la lande fleurie (scènes du pays de Carnac) ; Premier Impromptu ; Sérénade pour un soir de fête), quatre autres entre 1946 et $1956 \mathrm{chez}$ Durand (En carriole; Impromptu-Valse ; Une valse; Le Petit Train de Paimpol). Entre 1940 et 1943, comme déjà signalé à la note 34, que des chansons sous pseudonyme. 


\section{BIBLIOGRAPHIE}

a) Liste des "Variations... sans thèmes » d'Yves Margat dans Le Guide du concert citées dans l'article

Vol. 21 (1934-1935) :

$\mathrm{n}^{\circ} 3$ (19 octobre 1934), p. 45, «con bravura», http://1mhsbd.oicrm.org/media/ART-MAY-1934-02. pdf.

$\mathrm{n}^{\circ} 4$ (26 octobre 1934), p. 76, « risoluto».

$\mathrm{n}^{\circ} 5$ (2 novembre 1934), p. 108, " poco malinconico », http://lmhsbd.oicrm.org/media/ARTMAY-1934-03.pdf.

$\mathrm{n}^{\circ} 6$ (9 novembre 1934), p. 140, «simplice».

$\mathrm{n}^{\circ} 7$ (16 novembre 1934), p. 171, " mezza voce ", http://lmhsbd.oicrm.org/media/ARTMAY-1934-01.pdf.

$\mathrm{n}^{\circ} 8$ (23 novembre 1934), p. 205, « serioso ma non troppo".

no 9 (30 novembre 1934), p. 237, «innocente».

no 10 (7 décembre 1934), p. 266, " poco meno mosso ", http://lmhsbd.oicrm.org/media/ARTMAY-1934-04.pdf.

$\mathrm{n}^{\circ} 11$ (14 décembre 1934), p. 298, « con tenerezza».

$\mathrm{n}^{\circ} 16$ (18 janvier 1935), p. 394, «scherzando".

$\mathrm{n}^{\circ} 19$ (8 février 1935), p. 490, «con grazia».

$\mathrm{n}^{\circ} 20$ (15 février 1935), p. 522, «attacca subito l'allegro».

$\mathrm{n}^{\circ} 21$ (22 février 1935), p. 552, « quasi piano».

$\mathrm{n}^{\circ} 23$ (8 mars 1935), p. 618, «alla leggenda».

$\mathrm{n}^{\circ} 24$ (15 mars 1935), p. 650, « quasi tremolo».

Vol. 22 (1935-1936) :

no 1-2 (4 et 11 octobre 1935), p. 9, http://1mhsbd.oicrm.org/media/ART-MAY-1935-03.pdf.

$\mathrm{n}^{\circ} 4$ (25 octobre 1935), p. 73, http://1mhsbd.oicrm.org/media/ART-MAY-1935-04.pdf.

no 8 (22 novembre 1935), p. 203.

no 9 (29 novembre 1935), p. 234.

no 10 (6 décembre 1935), p. 266.

no 12-13-14 (20, 27 décembre 1935, 3 janvier 1936), p. 328.

$\mathrm{n}^{\circ} 15$ (10 janvier 1936), p. 364.

$\mathrm{n}^{\circ} 17$ (25 janvier 1936), p. 426.

no 19 (7 février 1936), p. 488.

$\mathrm{n}^{\circ} 20$ (14 février 1936), p. 522.

$\mathrm{n}^{\circ} 21$ (21 février 1936), p. 556.

$\mathrm{n}^{\mathrm{o}} 25$ (20 mars 1936), p. 680.

$\mathrm{n}^{\circ}$ 26-27-28 (27 mars, 3 et 10 avril 1936), p. 712.

$\mathrm{n}^{\circ} 33-34$ (15 et 22 mai 1936), p. 809.

Vol. 23 (1936-1937) :

n 3 (16 octobre 1936), p. 43, http://1mhsbd.oicrm.org/media/ART-MAY-1936-02.pdf. 
$\mathrm{n}^{\circ} 4$ (23 octobre 1936), p. 74.

no 5 (30 octobre 1936), p. 106, http://1mhsbd.oicrm.org/media/ART-MAY-1936-03.pdf.

$\mathrm{n}^{\circ} 7$ (13 novembre 1936), p. 170.

$\mathrm{n}^{\circ} 8$ (20 novembre 1936), p. 201, http://1mhsbd.oicrm.org/media/ART-MAY-1936-04.pdf.

no 9 (27 novembre 1936), p. 234.

no 10 (4 décembre 1936), p. 266, http://lmhsbd.oicrm.org/media/ART-MAY-1936-05.pdf.

n 11 (11 décembre 1936), p. 298, http://1mhsbd.oicrm.org/media/ART-MAY-1936-06.pdf.

n $12-13-14$ (18 et 25 décembre 1936, 1 janvier 1937), p. 329.

$\mathrm{n}^{\circ} 15$ (8 janvier 1937), p. 361.

$\mathrm{n}^{\circ} 16$ (15 janvier 1937), p. 393-394.

no 17 (22 janvier 1937), p. 426, http://1mhsbd.oicrm.org/media/ART-MAY-1937-01.pdf.

$\mathrm{n}^{\circ} 18$ (29 janvier 1937), p. 459, http://1mhsbd.oicrm.org/media/ART-MAY-1937-02.pdf.

$\mathrm{n}^{\circ} 19$ (5 février 1937), p. 491, http://1mhsbd.oicrm.org/media/ART-MAY-1937-03.pdf.

n 20 (12 février 1937), p. 522.

$\mathrm{n}^{\circ} 21$ (19 février 1937), p. 553.

$\mathrm{n}^{\circ} 22$ (26 février 1937), p. 588.

no 23 (5 mars 1937), p. 620, http://1mhsbd.oicrm.org/media/ART-MAY-1937-04.pdf.

no 27-28 (2 et 9 avril 1937), p. 681.

$\mathrm{n}^{\circ}$ 31-32 (30 avril, 7 mai 1937), p. 746.

$\mathrm{n}^{\circ} 35$ (28 mai 1937), p. 810.

Vol. 24 (1937-1938) :

$\mathrm{n}^{\circ} 1-2$ (1 et 8 octobre 1937), p. 11.

$\mathrm{n}^{\circ} 3$ (15 octobre 1937), p. 44

$\mathrm{n}^{\circ} 4$ (22 octobre 1937), p. 73.

$\mathrm{n}^{\mathrm{o}} 7$ (12 novembre 1937), p. 172, http://1mhsbd.oicrm.org/media/ART-MAY-1937-06.pdf.

no 8 (19 novembre 1937), p. 204, http://1mhsbd.oicrm.org/media/ART-MAY-1937-05.pdf.

no 10 (3 décembre 1937), p. 266.

$\mathrm{n}^{\circ} 11$ (10 décembre 1937), p. 298.

$\mathrm{n}^{\circ}$ 12-13-14 (17, 24, 31 décembre 1937), p. 331, http://1mhsbd.oicrm.org/media/ARTMAY-1937-07.pdf.

$\mathrm{n}^{\circ} 17$ (21 janvier 1938), p. 427.

$\mathrm{n}^{\circ} 18$ (28 janvier 1938), p. 462.

n 19 (4 février 1938), p. 491, http://1mhsbd.oicrm.org/media/ART-MAY-1938-01.pdf.

$\mathrm{n}^{\circ} 20$ (11 février 1938), p. 524, http://1mhsbd.oicrm.org/media/ART-MAY-1938-04.pdf.

$\mathrm{n}^{\circ} 23$ (4 mars 1938), p. 618, http://1mhsbd.oicrm.org/media/ART-MAY-1938-05.pdf.

$\mathrm{n}^{\circ}$ 28-29-30 (8, 15, 22 avril 1938), p. 745-746.

no 31-32 (29 avril, 6 mai 1938), p. 777-778.

Vol. 25 (1938-1939) :

no $1-2(7,14$ octobre 1938), p. 9. 
no 3 (21 octobre 1938), p. 44.

n 4 (28 octobre 1938), p. 76, http://1mhsbd.oicrm.org/media/ART-MAY-1938-02.pdf.

$\mathrm{n}^{\circ} 5$ (4 novembre 1938), p. 107.

$\mathrm{n}^{\circ} 6$ (11 novembre 1938), p. 140.

$\mathrm{n}^{\mathrm{o}} 7$ (18 novembre 1938), p. 172.

n 9 (2 décembre 1938), p. 236.

no 10 (9 décembre 1938), p. 267, http://1mhsbd.oicrm.org/media/ART-MAY-1938-03.pdf.

no 11-12-13 (16, 23, 30 décembre 1938), p. 300.

$\mathrm{n}^{\circ} 16$ (20 janvier 1939), p. 361, http://lmhsbd.oicrm.org/media/ART-MAY-1939-01.pdf.

no 20 (17 février 1939), p. 491.

no 21 (24 février 1939), p. 522, http://1mhsbd.oicrm.org/media/ART-MAY-1939-05.pdf.

no 25-26-27 (24 et 31 mars, 7 avril 1939), p. 652, http://lmhsbd.oicrm.org/media/ARTMAY-1939-02.pdf.

no 28-29 (14 et 21 avril 1939), p. 684, http://1mhsbd.oicrm.org/media/ART-MAY-1939-03.pdf.

n 32-33 (12 et 19 mai 1939), p. 746, http://lmhsbd.oicrm.org/media/ART-MAY-1939-04.pdf.

no 34 (26 mai 1939), p. 777.

\section{b) Autres écrits de ou sur Margat}

Margat, Yves (1936), Exercices préparatoires à l'étude de l'harmonie (1'e série), Paris, Durand.

Margat, Yves (1937), Réalisations des Exercices préparatoires à l'étude de l'harmonie (1'e série), Paris, Durand.

Margat, Yves (1939), Exercices préparatoires à l'étude de l'harmonie (2e série), Paris, Durand.

Margat, Yves (1941), Traité de l'harmonie classique, Paris, Durand.

Margat, Yves (1951), Réalisations des exercices, basses et chants du Traité de l'harmonie classique, Paris, Durand.

Margat, Yves (1953), Cours pratique d'harmonisation et d'accompagnement au piano, précédé d'un Abrégé de l'harmonie classique, Paris, Durand. Une version numérisée est disponible pour la consultation en ligne : https://fr.scribd.com/document/59132824/2069221-Cours-pratique-daccompagnementau-piano-Yves-Margat

P. V. (1936), «Interview. Yves Margat », Le Guide du concert, vol. 23, n 5, 30 octobre, p. 98.

Sirinelli, Jean-François (dir.) (1993), La France de 1914 à nos jours, Paris, Presses universitaires de France.

X. (1935), «Un cours d'harmonie avec... Yves Margat », Le Guide du concert, vol. 21, n 27-28-29, 5-12-19 avril, p. 707.

X. (1937), «Interview avec Yves Margat », Le Guide du concert, vol. 24, nº 4, 22 octobre, p. 67.

c) Autres références

[Anonyme] (1930a), "Une innovation intéressante. La TSF dans le train », Le Petit Journal, 22 janvier, $\mathrm{n}^{\circ}$ 24478, p. 2. Disponible sur Gallica : http://gallica.bnf.fr/ark:/12148/bpt6k6320375/f2.

[Anonyme] (1930b), «Légion d'honneur », Le Matin, 30 juillet, n 16933, p. 4. Disponible sur Gallica : http://gallica.bnf.fr/ark:/12148/bpt6k576964q/f4.

[Anonyme] (1936), "Une enquête. Où va la musique ? ", Le Guide du concert, vol. 23, n 1-2 (2 et 9 octobre), p. 30. Disponible dans la banque de données du PHEM : http://1mhsbd.oicrm.org/ media/ART-ANO-1936-01.pdf. 
Barraud, Henri (1938), «La musique allemande depuis Hindemith », Le Guide du concert, vol. 24, n 15 (7 janvier), p. 360-362. Disponible dans la banque de données du PHEM : http://lmhsbd.oicrm.org/ media/ART-BAH-1938-01.pdf.

Bender, Gabriel (1913), "Éducation sensorielle », Le Guide du concert, vol. 5, nº 10 (13 décembre), p. 145. Disponible dans la banque de données du PHEM : http://lmhsbd.oicrm.org/media/ARTBEG-1913-01.pdf.

G. B. [Gabriel Bender] (1930), "Une enquête. Audition directe et audition indirecte ", Le Guide du concert, vol. 17, $\mathrm{n}^{\circ} 1$ (3 octobre), p. 9-10. Disponible dans la banque de données du PHEM : http:// lmhsbd.oicrm.org/media/ART-BEG-1930-01.pdf.

G. B. [Gabriel Bender] (1936), « Notes brèves », vol. 22, n 20, 14 février, p. 515.

Bender, Gabriel et Marc Rousseau (1928), «Entretiens sur le plaisir musical [14] », Le Guide du concert, vol. $15, \mathrm{n}^{\circ} 2$ (12 octobre), p. 27. Disponible dans la banque de données du PHEM : http://1mhsbd. oicrm.org/media/ART-BEG-1928-01.pdf.

Berstein, Serge (1982), Histoire du Parti radical, vol. 2 : " Crise du radicalisme, 1926-1939 ", Paris, Presses de Sciences Po.

Berstein, Serge (1988), La France des années 1930, Paris, Armand Colin.

Berstein, Serge (2003), « Le renouvellement du Parti radical dans les années 1930. Un renouveau de la gauche? ", dans Antoine Prost (dir.), Jean Zay et la gauche du radicalisme, Paris, Presses de Sciences Po, p. 83-97.

Brunel, Raoul (1937), "Où va la musique ? ", Le Ménestrel, $1^{\text {re }}$ partie : vol. 99, no 13/5265 (26 mars) ; $2^{\mathrm{e}}$ partie : vol. 99, $\mathrm{n}^{\mathrm{o}}$ 14-15/5266-5267 (2 et 9 avril 1937). Disponible dans la banque de données du PHEM : http://1mhsbd.oicrm.org/media/ART-BLR-1937-01.pdf, http://lmhsbd.oicrm.org/ media/ART-BLR-1937-02.pdf.

Buch, Esteban (2006), Le cas Schönberg. Naissance de l'avant-garde musicale, Paris, Gallimard/NRF.

Cochet, François (dir.) (2008), De Gaulle et les « Jeunes Turcs» dans les armées occidentales (1930-1945). Une génération de la réflexion à l'action, Paris, Riveneuve.

Heu, Pascal Manuel (2005), À la recherche du patriarche perdu. La critique, le prospecteur d'arts : Emile Vuillermoz (1878-1960) et le cinéma, 3 vol., thèse de doctorat, Université de Paris I Panthéon-Sorbonne.

Ferchauld, Guy (1938), «Le drame wagnérien. Sa genèse, sa nature et ses réalisations scéniques dans l'Allemagne actuelle » [série de 5 articles], Le Guide du concert, vol. 24, $\mathrm{n}^{\text {os }} 16-20$ (14, 21, 28 janvier et 4, 11 février 1938), p. 391-394, 425-427, 455-459, 487-491, 491, 519-523. Disponible dans la banque de données du PHEM : http://1mhsbd.oicrm.org/media/ART-FEG-1938-01.pdf, http://1mhsbd. oicrm.org/media/ART-FEG-1938-02.pdf, http://1mhsbd.oicrm.org/media/ART-FEG-1938-03. pdf, http://1mhsbd.oicrm.org/media/ART-FEG-1938-04.pdf, http://1mhsbd.oicrm.org/media/ ART-FEG-1938-05.pdf.

Jarrige, François ([2014]2016), Technocritiques. Du refus des machines à la contestation des technosciences, $2^{\mathrm{e} e ́ d ., ~ P a r i s, ~ L a ~ D e ́ c o u v e r t e . ~}$

Kalifa, Dominique (2017), La véritable histoire de la "Belle Époque», Paris, Fayard.

Kinsky, Georg et Hans Halm (2014), Ludwig van Beethoven. Thematisch-bibliographisches Werkverzeichnis, $2^{e}$ éd. en 2 vol., München, Henle.

Lazzaro, Federico (2011), Danza, incantesimo e preghiera. Il "rinnovamento espressivo " nella musica francese degli anni '30 del Novecento, thèse de doctorat, Università degli studi di Pavia. Accessible en ligne : https://www.academia.edu/1884895/PhD diss. Pavia University 2011 Danza incantesimo e preghiera. Il rinnovamento espressivo nella musica francese degli anni 30 del Novecento.

Lazzaro, Federico (2017), « Le discours esthétique dans la presse musicale française. Cadre théorique et méthodologie du projet de recherche Histoire de l'esthétique musicale en France, 1900-1950 (PHEM) ", Revue musicale OICRM, vol. 4, $\mathrm{n}^{\circ} 2$ (novembre 2017) : " Enjeux culturels dans la presse musicale française, 1900-1925 ", p. 163-175, http://revuemusicaleoicrm.org/rmo-vol4-n2/discoursesthetique-presse-musicale/. 
Lazzaro, Federico (sous presse), Écoles de Paris en musique, 1920-1950. Identités, nationalisme, cosmopolitisme, Paris, Vrin.

Meyer, Leonard B. ([1956]2011), Émotion et signification en musique, traduit de l'anglais par Catherine Delaruelle, Arles, Actes Sud.

Moore, Christopher (2006), Music in France and the Popular Front (1934-1938). Politics, Aesthetics and Reception, thèse de doctorat, McGill University, 2006.

Moore, Christopher (2008), "Socialist Realism and the Music of the French Popular Front ", The Journal of Musicology, vol. 25, n 4 (octobre), p. 473-502.

[Nourry, Pierre] (1937), "Le jazz hot », Le Guide du concert, vol. 24, n 3 (15 octobre), p. 54. Disponible dans la banque de données du PHEM : http://lmhsbd.oicrm.org/media/ART-NOP-1937-01.pdf.

Spire, Florence (1997), L'adaptation française de la méthode Kodály, mémoire de maîtrise, Université Paris-Sorbonne.

Tiersot, Julien (1927), « Manuscrits de Beethoven », Revue de musicologie, vol. 8, no 22 (mai), p. 65-76.

Vigreux, Jean (2016), Histoire du Front populaire. L'échappée belle, Paris, Tallandier.

Virneisel, Wilhelm (1966), "Zur Handschrift des Missa solemnis von Beethoven ", Österreichische Musikzeitschrift, vol. 21, p. 261-268.

Winter, Robert (1984), "Reconstructing Riddles. The Sources for Beethoven's Missa solemnis ", dans Lewis Lockwood et Phyllis Benjamin (dir.), Beethoven Essays. Studies in Honor of Elliot Forbes, Cambridge, Harvard University Press, p. 217-250. 